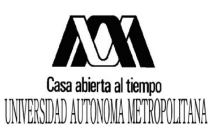

\section{UNIVERSIDAD AUTÓNOMA METROPOLITANA UNIDAD IZTAPALAPA \\ DIVISIÓN DE CIENCIAS BÁSICAS E INGENIERÍA}

\title{
GRUPOS PARATOPOLÓGICOS
}

\author{
Tesis que presenta \\ Iván Sánchez Romero \\ Para obtener el grado de \\ Doctor en Matemáticas
}

Asesor de tesis: Dr. Mikhail Tkachenko

Sinodales:

Dr. Richard Gordon Wilson Roberts

Dr. Vladimir Tkachuk Vladimirovich

Dr. Mikhail Tkachenko Gelievich

Dr. Salvador García Ferreira

Dr. Manuel Sanchis López

México, D.F., 10 de Diciembre de 2013 


\section{GRUPOS PARATOPOLÓGICOS}

Tesis que presenta

Iván Sánchez Romero

Para obtener el grado de

Doctor en Matemáticas

Asesor de tesis: Dr. Mikhail Tkachenko

Sinodales:

Dr. Richard Gordon Wilson Roberts

Dr. Vladimir Tkachuk Vladimirovich

Dr. Mikhail Tkachenko Gelievich

Dr. Salvador García Ferreira

Dr. Manuel Sanchis López

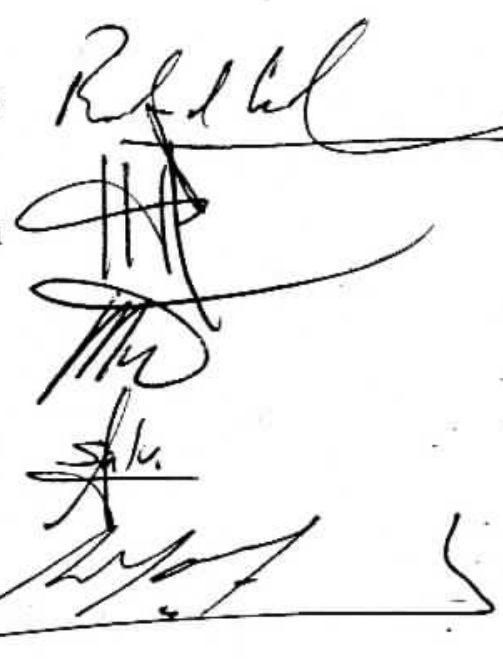

México, D.F., 10 de Diciembre de 2013 


\section{Índice general}

Resumen II

Introducción

1. Preliminares 1

1.1. Definiciones y ejemplos . . . . . . . . . . . . . . . . 1

1.2. Propiedades básicas . . . . . . . . . . . . . . . . . . . . 2

1.3. Grupo topológico asociado . . . . . . . . . . . . 5

1.4. Regularización . . . . . . . . . . . . . . . . . . 7

2. Encajes $\quad 8$

2.1. Número de simetría . . . . . . . . . . . . . . . . 8

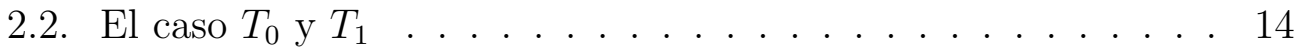

3. Funciones cardinales $\quad 28$

3.1. Índice de regularidad y de estrechez . . . . . . . . . . . . . . . 28

3.2. El rango de la diagonal . . . . . . . . . . . . . . . . . . . 38

3.3. Condensaciones . . . . . . . . . . . . . . . . . . . . 45

4. Conclusiones 49

4.1. Problemas abiertos . . . . . . . . . . . . . . 49 


\section{Resumen}

En el primer capítulo se encuentran algunas definiciones y resultados que utilizaremos en los capítulos posteriores.

En el segundo capítulo de esta tesis damos una caracterización interna de los subgrupos de productos de grupos paratopológicos $T_{i}$ primero (segundo) numerables, para $i=0,1$. En el caso $T_{1}$ demostramos que un grupo paratopológico $T_{1} G$ es topológicamente isomorfo a un subgrupo de un producto de grupos paratopológicos $T_{1}$ primero (segundo) numerables si y sólo si $G$ es $\omega$-balanceado (totalmente $\omega$-estrecho) y $G$ tiene número de simetría numerable, donde el número de simetría de un grupo semitopológico es definido como el mínimo cardinal $\kappa$ tal que para cada vecindad $U$ de $e$ en $G$, existe una familia $\gamma \subseteq \mathcal{N}(e)$ tal que $\bigcap_{V \in \gamma} V^{-1} \subseteq U$ y $|\gamma| \leq \kappa$. También probamos que todo grupo paratopológico 2-pseudocompacto con número de simetría numerable, es un grupo topológico.

Construimos un grupo paratopológico abeliano $H$, funcionalmente Hausdorff, totalmente $\omega$-estrecho con pseudocarácter numerable que no se puede condensar en un grupo paratopológico Hausdorff primero numerable. Además, $H s(H)>\omega$. Este ejemplo responde en forma negativa algunas preguntas planteadas por M. Sanchis y M. Tkachenko.

En la sección 3.1 probamos que un grupo paratopológico regular totalmente $\omega$-estrecho $G$ tiene índice de regularidad numerable (lo cual responde una pregunta publicada por M. Tkachenko), es decir, para cada vecindad $U$ de la identidad $e$ en $G$, podemos encontrar una vecindad $V$ de $e$ y una familia numerable $\gamma$ de vecindades de $e$ tales que $\bigcap_{W \in \gamma} V W^{-1} \subseteq U$. Utilizando este hecho demostramos que todo grupo paratopológico regular (Hausdorff) totalmente $\omega$-estrecho es completamente regular (funcionalmente Hausdorff). También probamos que en todo grupo paratopológico regular $G$, el índice de regularidad de $G$ es menor o igual que el número Lindelöf débil de $G$.

En la sección 3.2 se demuestra que todo grupo paratopológico Hausdorff con $\pi$-carácter numerable tiene una diagonal $G_{\delta}$ de rango infinito. Este resultado responde en forma positiva una pregunta planteada por A. Arhangel'skii y A. Bella. También construimos un grupo paratopológico Hausdorff de $\pi$-carácter no numerable el cual contiene un subgrupo denso segundo numerable.

En la sección 3.3 probamos que todo grupo paratopológico Hausdorff Lindelöf con pseudocarácter numerable se puede condensar sobre un espacio metrizable separable. Este resultado responde una pregunta de Tkachenko. 


\section{Introducción}

Un grupo semitopológico (paratopológico) $(G, \tau)$ consiste de un grupo abstracto $G$ dotado de una topología $\tau$ con la cual la multiplicación es separadamente (conjuntamente) continua. Un grupo semitopológico (paratopológico) es llamado cuasitopológico (topológico) si la inversión es continua. Dado un grupo semitopológico $G$ con identidad $e$, denotemos por $\mathcal{N}_{G}(e)$ a la familia de vecindades abiertas de $e$ en $G$. Si no hay ambigüedad en cuanto al grupo semitopológico en cuestión, solamente escribiremos $\mathcal{N}(e)$.

Durante mucho tiempo se ha investigado qué condiciones topológicas y/o algebraicas en un grupo semitopológico (paratopológico) $G$ implican que $G$ es un grupo topológico. En 1957 R. Ellis [13] demostró que cada grupo semitopológico localmente compacto Hausdorff es un grupo topológico. Después, en 1960 Żelazko [35] prueba que cada grupo semitopológico completamente metrizable es un grupo topológico. Bouziad en [11] demuestra que cada grupo semitopológico Čech-completo es un grupo topológico.

En 2007 M. Sanchis y M. Tkachenko demuestran que cada $P$-grupo paratopológico Hausdorff Lindelöf es un grupo topológico [30]. En la proposición 2.1.8 debilitamos la condición de espacio Hausdorff a $T_{1}$. En 2010 Ravsky prueba que en todo grupo paratopológico 2-pseudocompacto de pseudocarácter numerable la inversa es continua [24], respondiendo de esta forma a una pregunta planteada por Alas y Sanchis en [1]. En la sección 2.1, en la proposición 2.1.11 damos una caracterización de cuándo un grupo paratopológico 2-pseudocompacto es un grupo topológico, la cual generaliza el resultado de Ravsky mencionado anteriormente.

Katz demuestra que un grupo topológico es topológicamente isomorfo a un subgrupo de un producto de grupos topológicos primero numerables (metrizables) si y sólo si es $\omega$-balanceado [18]. El caso de grupos topológicos segundo numerables es descrito por Guran en [15]: un grupo topológico $G$ admite un encaje topológico como un subgrupo de un producto de grupos topológicos segundo numerables si y sólo si $G$ es $\omega$-estrecho.

Sea $\mathcal{P}$ una propiedad topológica y/o algebraica. Decimos que un grupo paratopológico (topológico) $G$ es proyectivamente $\mathcal{P}$ si para cada $U \in \mathcal{N}_{G}\left(e_{G}\right)$, existe un homomorfismo continuo $p: G \rightarrow H$ sobre un grupo paratopológico (topológico) $H$ con la propiedad $\mathcal{P}$ tal que $p^{-1}(V) \subseteq U$, para alguna $V \in \mathcal{N}_{G}\left(e_{H}\right)$. Por el corolario 2.2.2, un grupo paratopológico es proyecti-

vamente $T_{i}$ primero (segundo) numerables si y sólo si es topológicamente isomorfo a un subgrupo de un producto de grupos paratopológicos $T_{i}$ prime- 
ro (segundo) numerables, para $i=0,1,2,3,3 \frac{1}{2}$.

Tkachenko utiliza el concepto de número de Hausdorff, para caracterizar los grupos paratopológicos que son proyectivamente Hausdorff primero (segundo) numerables ([32, Theorems 2.7-2.8]). En ese mismo artículo Tkachenko describe el caso de grupos paratopológicos regulares: un grupo paratopológico $G$ admite un encaje topológico como un subgrupo de un producto de grupos paratopológicos primero (segundo) numerables si y sólo si $G$ tiene indice de regularidad numerable y es $\omega$-balanceado (totalmente $\omega$-estrecho).

En la sección 2.2, en los teoremas 2.2.8 y 2.2.10 mostramos que un grupo paratopológico $T_{0}$ es proyectivamente $T_{0}$ primero (segundo) numerable si y sólo si es $\omega$-balanceado (totalmente $\omega$-estrecho). En los teoremas 2.2.11 y 2.2.14 describimos el caso de grupos paratopológicos $T_{1}$, concretamente, probamos que un grupo paratopológico $T_{1} G$ es topológicamente isomorfo a un subgrupo de un producto de grupos paratopológicos $T_{1}$ primero (segundo) numerables si y sólo si $G$ es $\omega$-balanceado (totalmente $\omega$-estrecho) y $G$ tiene número de simetría numerable. Un grupo paratopológico $G$ tiene número de simetría numerable si para cada vecindad $U$ de $e$ en $G$, existe una familia numerable $\gamma \subseteq \mathcal{N}(e)$ tal que $\bigcap_{V \in \gamma} V^{-1} \subseteq U$.

En [30, Problem 6.3], Sanchis y Tkachenko preguntan ¿todo grupo paratopológico Hausdorff totalmente $\omega$-estrecho con pseudocarácter numerable se puede condensar en un grupo paratopológico $H$ Hausdorff segundo numerable? El ejemplo 2.2.22 de esta tesis responde en forma negativa a esta pregunta ya que construimos un grupo paratopológico Hausdorff totalmente $\omega$-estrecho con pseudocarácter numerable que no admite un isomorfismo continuo sobre un grupo paratopológico Hausdorff segundo numerable. El grupo $H$ del ejemplo 2.2.22 tiene número de Hausdorff no numerable lo cual responde (en forma negativa) la pregunta planteada por Tkachenko en [32, Problem 4.1]: ¿cada grupo paratopológico Hausdorff totalmente $\omega$-estrecho $G$ tiene número de Hausdorff numerable? De esta forma, el ejemplo 2.2.22 es uno de los resultados más importantes de este trabajo.

En la sección 3.1, demostramos algunas desigualdades en la clase de grupos paratopológicos (semitopológicos). En particular, probamos que todo grupo paratopológico regular totalmente $\omega$-estrecho $G$ tiene índice de regularidad numerable (ver Teorema 3.1.3). Utilizando el Teorema 3.1.3 y [32, Theorem 3.8] podemos concluir que un grupo paratopológico regular $G$ es topológicamente isomorfo a un subgrupo de un producto de grupos paratopológicos regulares segundo numerables si y sólo si $G$ es totalmente $\omega$ estrecho. Este hecho responde en forma positiva a un problema formulado 
por M. Tkachenko (ver [34]): supongamos que $G$ es un grupo paratopológico Tychonoff totalmente $\omega$-estrecho. ¿Es $G$ topológicamente isomorfo a un subgrupo de un producto de grupos paratopológicos regulares segundo numerables? Por esta razón, el teorema 3.1.3 es uno de los resultados principales de la tesis.

El siguiente problema aún permanece abierto: supongamos que $G$ es un grupo paratopológico regular (primero numerable). ¿Es $G$ completamente regular?, ¿es $G$ funcionalmente Hausdorff? En esta dirección probamos que todo grupo paratopológico regular (Hausdorff) totalmente $\omega$-estrecho es completamente regular (funcionalmente Hausdorff).

En [32] se demuestra que en todo grupo paratopológico regular el número de Lindelöf es mayor o igual que el índice de regularidad. Claramente el número Lindelöf débil de un espacio $X$, denotado por $w l(X)$ es menor o igual que el número de Lindelöf de $X$. El teorema 3.1.12 establece que para todo grupo paratopológico regular $G$ se satisface la desigualdad $\operatorname{Ir}(G) \leq w l(G)$. En particular, cada grupo paratopológico regular precompacto (separable) tiene índice de regularidad numerable. No sabemos si todo grupo paratopológico regular $\omega$-estrecho tiene índice de regularidad numerable (ver problema 3.1.4).

En [26] A. Ravsky prueba que todo grupo paratopológico $G$ cumple la igualdad $\pi \omega(G)=\operatorname{In}_{l}(G) \pi \chi(G)$. Recientemente, en [20] Li, Mou y Wang (A. Ravsky de forma independiente) demuestran la desigualdad $d(G) \leq$ $\operatorname{In}_{l}(G) \chi(G)$ para un grupo semitopológico $G$. En el teorema 3.1.24 demostramos que dado un grupo semitopológico $G$ se cumple $\pi \omega(G)=I n_{l}(G) \pi \chi(G)$. Claramente, el teorema 3.1.24 generaliza los resultados mencionados anteriormente.

En la sección 3.2, probamos resultados relacionados con el rango de la diagonal de un grupo paratopológico. En [21], C. Liu demuestra que todo grupo paratopológico $G$ Hausdorff primero numerable tiene una digonal $G_{\delta}$ regular. Posteriormente, A. Arhangel'skii y A. Bella prueban que todo grupo paratopológico Hausdorff primero numerable tiene una digonal $G_{\delta}$ de rango infinito. Generalizando de esta forma el resultado de C. Liu debido a que un espacio con una diagonal $G_{\delta}$ de rango 3 tiene una diagonal $G_{\delta}$ regular (ver [2, Proposition 1]).

En [2], A. Arhangel'skii y A. Bella plantean el problema: ¿Todo grupo paratopológico Hausdorff (regular) con $\pi$-carácter numerable tiene una diagonal $G_{\delta}$ de rango infinito? El teorema 3.2.8, uno de los más importantes de la tesis, responde en forma positiva esta pregunta. También demostramos 
que si $G$ es un grupo paratopológico Hausdorff $\omega$-balanceado con número de Hausdorff numerable y $\psi(G) \leq \omega$, entonces $G$ tiene una diagonal $G_{\delta}$ de rango infinito (ver proposición 3.2.16). Este hecho generaliza el resultado de A. Arhangel'skii y A. Bella mencionado anteriormente, porque todo grupo paratopológico Hausdorff primero numerable es $\omega$-balanceado, tiene número de Hausdorff numerable y pseudocarácter numerable.

En la sección 3.3, trabajamos con condensaciones de un grupo paratopológico sobre un espacio segundo numerable. Basándonos en los artículos [3] y [29], probamos que un grupo semitopológico completamente regular $G$ $\omega$-estrecho con número de Hausdorff numerable y $\psi(G) \leq \omega$ se puede condensar sobre un espacio metrizable segundo numerable (ver teorema 3.3.1). Apoyándonos en este resultado, demostramos que todo grupo paratopológico Hausdorff Lindelöf con pseudocarácter numerable admite una subtopología metrizable segundo numerable, lo cual responde una pregunta planteada por M. Tkachenko en [33] (ver teorema 3.3.4).

Se prueba que todo grupo semitopológico (paratopológico) regular (Hausdorff) $\omega$-estrecho con número de Hausdorff numerable y pseudocarácter numerable se puede condensar sobre un espacio Urysohn segundo numerable (ver teorema 3.3.8 y corolario 3.3.10). 


\section{Capítulo 1}

\section{Preliminares}

\subsection{Definiciones y ejemplos}

Un grupo semitopológico $(G, \tau)$ consiste de un grupo algebraico $G$ dotado de una topología $\tau$ con la cual la multiplicación en $G$ es separadamente continua. El siguiente resultado es una consecuencia inmediata de la definición de grupo semitopológico.

Proposición 1.1.1. Si $G$ es un grupo semitopológico y $A, O \subseteq G$ con $O$ abierto en $G$, entonces los conjuntos $A O=\{a o: a \in A, o \in O\}$ y $O A=$ $\{$ oa: $o \in O, a \in A\}$ también son abiertos en $G$.

Dado un grupo $G$, la función "inversión" In: $G \rightarrow G$ está definida como $\operatorname{In}(x)=x^{-1}$, para cada $x \in G$. Un grupo semitopológico es llamado grupo cuasitopológico si la inversión es continua.

Un grupo paratopológico $(G, \tau)$ consiste de un grupo algebraico $G$ dotado de una topología $\tau$ con la cual la multiplicación en $G$ es continua. Claramente, todo grupo paratopológico es un grupo semitopológico.

Ejemplo 1.1.2. Sea $\mathbb{S}$ la recta de Sorgenfrey, es decir, los números reales $\mathbb{R}$ con la suma y la topología $\tau$ generada por los intervalos $[a, b)$, donde $a, b \in \mathbb{R}$ y $a<b$. Entonces $\mathbb{S}$ es un grupo paratopológico. No obstante, la inversión en $\mathbb{S}$ no es continua.

Un grupo paratopológico es llamado grupo topológico si la inversión es continua. 
Sea $G$ un grupo. Fijemos $g \in G$. Consideremos las funciones $\lambda_{g}$ y $\rho_{g}$, ambas de $G$ en $G$, definidas como $\lambda_{g}(x)=g x$ y $\rho_{g}(x)=x g$ para cada $x \in G$. La función $\lambda_{g}$ (respectivamente, $\rho_{g}$ ) es llamada la traslación izquierda (respectivamente, traslación derecha) de $G$ por $g$. Claramente $G$ es un grupo semitopológico si y sólo si las funciones $\lambda_{g}$ y $\rho_{g}$ son continuas para cada $g \in G$.

Proposición 1.1.3. Sea $G$ un grupo semitopológico y g un elemento de $G$. Entonces,

a) las funciones $\lambda_{g}$ y $\rho_{g}$ son homeomorfismos del espacio $G$ en él mismo;

b) para cualquier base local $\mathcal{B}_{e}$ de e en $G$, las familias $\left\{g U: U \in \mathcal{B}_{e}\right\}$ y $\left\{U g: U \in \mathcal{B}_{e}\right\}$ son una base local de $g$ en $G$.

Demostración. Probemos a). Como $G$ es un grupo semitopológico, las traslaciones $\lambda_{g}$ y $\rho_{g}$ son continuas. Es fácil ver que la inversa de $\lambda_{g}$ es $\lambda_{g^{-1}}$, por lo tanto, $\lambda_{g}$ es un homeomorfismo de $G$ en $G$. De forma similar se demuestra que $\rho_{g}$ es un homeomorfismo de $G$ en él mismo. La parte b) se sigue de a).

Corolario 1.1.4. Todo grupo semitopológico es un espacio homogéneo.

\subsection{Propiedades básicas}

En [23], Pontryagin caracteriza internamente cuándo un grupo algebraico $G$ dotado de una topología $\tau$ es un grupo topológico. Haciendo una pequeña modificación en el resultado de Pontryagin se obtiene en grupos paratopológicos lo siguiente.

Teorema 1.2.1. ([25]) Sean $G$ un grupo paratopológico y $\mathcal{U}$ una base local de la identidad e en $G$. Entonces:

i) para $U, V \in \mathcal{U}$, existe un elemento $W \in \mathcal{U}$ el cual satisface $W \subseteq U \cap V$;

ii) para cada $U \in \mathcal{U}$ y cada $x \in U$, existe $V \in \mathcal{U}$ tal que $V x \subseteq U$;

iii) para cada $U \in \mathcal{U}$ y cada $x \in G$, existe $V \in \mathcal{U}$ tal que $x V x^{-1} \subseteq U$;

iv) para cada $U \in \mathcal{U}$, existe un elemento $V \in \mathcal{U}$ el cual satisface $V^{2} \subseteq U$. 
Recíprocamente, sean $G$ un grupo y $\mathcal{U}$ una familia de subconjuntos de $G$ que satiface las condiciones i)-iv). Entonces $\mathcal{B}_{\mathcal{U}}=\{U a: a \in G, U \in \mathcal{U}\}$ es una base para una topología $\tau_{\mathcal{U}}$ en $G$ tal que $\left(G, \tau_{\mathcal{U}}\right)$ es un grupo paratopológico. Además, la familia $\{a U: a \in G, U \in \mathcal{U}\}$ también es una base para la topología $\tau_{\mathcal{U}}$.

Demostración. Supongamos que $G$ es un grupo paratopológico. La parte i) se cumple porque $\mathcal{U}$ es una base local $e$. Los incisos ii) y iii) se siguen de la continuidad de las funciones $\rho_{x}$ y $\rho_{x^{-1}} \circ \lambda_{x}$. Como la multiplicación es continua en $G$, se satisface iv).

Probemos el recíproco. Sea $\mathcal{U}$ una familia de subconjuntos de $G$ que satiface las condiciones i)-iv). Sea $\tau$ la familia de conjuntos $W \subseteq G$ que satisfacen la siguiente condición:

( $\star$ para cada $x \in W$, existe $U \in \mathcal{U}$ tal que $U x \subseteq W$.

Afirmación 1. $\tau$ es una topología en $G$.

En efecto, es claro que el vacío y $G$ están en $\tau$. También es claro que la unión arbitraria de elementos de $\tau$ está en $\tau$. Ahora, tomemos $W_{1}, W_{2} \in \tau$. Pongamos $W=W_{1} \cap W_{2}$. Veamos que $W$ pertenece a $\tau$. Tomemos $x \in W$, existen $U_{1}, U_{2} \in \mathcal{U}$ tales que $U_{i} x \subseteq W_{i}$ para $i=1,2$. Por i), podemos encontrar $U \in \mathcal{U}$ tal que $U \subseteq U_{1} \cap U_{2}$. Por lo tanto, $U x \subseteq W_{1} \cap W_{2}=W$. Se sigue de lo anterior que $W \in \tau$. Hemos probado la afirmación.

Afirmación 2. $U x \in \tau$, para cada $x \in G$ y cada $U \in \mathcal{U}$.

Tomemos $y \in U x$, equivalentemente, $y x^{-1} \in U$. Por ii), existe un elemento $V \in \mathcal{U}$ el cual satisface $V y x^{-1} \subseteq U$, es decir, $V y \subseteq U x$. Por lo tanto, el conjunto $U x$ está en $\mathcal{U}$.

La afirmación 2 y la condición ii) implican lo siguiente:

Afirmación 3. La familia $\mathcal{B}_{\mathcal{U}}=\{U a: a \in G, U \in \mathcal{U}\}$ es una base para la topología $\tau$. En consecuencia $\tau=\tau_{\mathcal{U}}$.

Afirmación 4. La multiplicación en $G$ es continua respecto a la topología $\tau$.

Tomemos $a, b \in G$ y $O$ un elemento de $\tau$ tal que $a b \in O$. Por la definición de $\tau$, podemos encontrar $W \in \mathcal{U}$ tal que $W a b \subseteq O$. La condición iv) garantiza la existencia de un elemento $U \in \mathcal{U}$ el cual satisface $U^{2} \subseteq W$. Por iii), existe $V \in \mathcal{U}$ tal que $a V a^{-1} \subseteq U$. Se sigue de lo anterior que $U\left(a V a^{-1}\right) \subseteq U^{2} \subseteq W$, por lo tanto, $U a V b=U\left(a V a^{-1}\right) a b \subseteq W a b$. Queda probada la afirmación. 
Afirmación 5. $b V \in \tau$, para cada $b \in G$ y cada $V \in \mathcal{U}$.

Tomemos $y \in b V$, es decir, $b^{-1} y \in V$. Por ii), existe $W \in \mathcal{U}$ tal que $W b^{-1} y \subseteq V$. Aplicando iii), podemos encontrar un elemento $U \in \mathcal{U}$ el cual satisface $b^{-1} U b \subseteq W$. En consecuencia, $b^{-1} U y=\left(b^{-1} U b\right) b^{-1} y \subseteq W b^{-1} y \subseteq V$, esto es, $b^{-1} U y \subseteq V$. Por lo tanto, $U y \subseteq b V$. Hemos probado la afirmación.

Por último, de la afirmación 5 y la condición iii) se concluye que la familia $\{a U: a \in G, U \in \mathcal{U}\}$ también es una base para la topología $\tau$.

Si $A$ es un subconjunto de un grupo semitopológico $G$, definimos el inverso de $A$ como $A^{-1}=\left\{a^{-1}: a \in A\right\}$. Decimos que $A$ es simétrico si $A=A^{-1}$.

Proposición 1.2.2. Sea $G$ un grupo paratopológico y $\mathcal{U}$ una base local de la identidad e en $G$. Entonces son equivalentes:

a) G es un grupo topológico;

b) para cada $U \in \mathcal{U}$, existe $V \in \mathcal{U}$ tal que $V^{-1} \subseteq U$.

Demostración. a) implica b) porque la inversión es continua en la identidad. Probemos que b) implica a). Tomemos un abierto $O$ en $G$ y un elemento arbitrario $o \in O$. El conjunto $o^{-1} O$ es una vecindad abierta de $e$. Como $\mathcal{U}$ es una base local de $e$, podemos encontrar $U \in \mathcal{U}$ tal que $U \subseteq o^{-1} O$. Por hipótesis, existe un elemento $V \in \mathcal{U}$ el cual satisface $V^{-1} \subseteq U$. Por lo tanto, $V \subseteq U^{-1} \subseteq O^{-1} o$, en consecuencia, $o^{-1} \in V o^{-1} \subseteq O^{-1}$. Como $G$ es un grupo paratopológico, $V o^{-1}$ es una vecindad abierta de $o^{-1}$. Por lo tanto, el conjunto $O^{-1}$ es abierto en $G$. Hemos demostrado que la inversión es continua en $G$.

Ejemplo 1.2.3. Consideremos el grupo multiplicativo $\mathcal{S}^{1}=\{x \in \mathbb{C}:|x|=$ 1\}. Sea $U_{n}=\left\{e^{i \theta}: 0 \leq \theta<\frac{1}{n}\right\}$ para cada $n \in \mathbb{N}$. Entonces existe una topología $\tau$ en $\mathcal{S}^{1}$ tal que $\mathbb{K}=\left(\mathcal{S}^{1}, \tau\right)$ es un grupo paratopológico y la familia $\mathcal{U}=\left\{U_{n}: n \in \mathbb{N}\right\}$ es una base local de la identidad. Sin embargo, $\mathbb{K}$ no es un grupo topológico. El grupo paratopológico $\mathbb{K}$ es conocido como la circunferencia de Sorgenfrey.

Demostración. Probemos que la familia $\mathcal{U}$ satisface las condiciones del teorema 1.2.1. Las condiciones i) y iii) son claras. Demostremos ii). Para ello, tomemos $U_{n} \in \mathcal{U}$ y $x \in U_{n}$. Por la definición de $U_{n}, x=e^{i \theta}$ con $0 \leq$ 
$\left.\theta<\frac{1}{n}\right\}$. Podemos encontrar $m \in \mathbb{N}$ tal que $\frac{1}{m}<\frac{1}{n}-\theta$. Si $y \in U_{m}$, entonces $y x=e^{i \alpha}$ con $\alpha \in\left[0, \frac{1}{n}\right)$, es decir, $U_{m} x \subseteq U_{n}$. La parte iv) es consecuencia de $U_{n}^{2}=\left\{e^{i \theta}: 0 \leq \theta<\frac{2}{n}\right\}$. De acuerdo a la proposición 1.2.2, $\mathbb{K}$ no es un grupo topológico porque ningún elemento de $\mathcal{U}$ está contenido en $U_{1}^{-1}=\left\{e^{-i \theta}: 0 \leq \theta<1\right\}$.

Definición 1.2.4. Dado un grupo semitopológico $G$ con identidad e, denotemos por $\mathcal{N}_{G}($ e) a la familia de vecindades abiertas de e en G. Si no hay ambigüedad en cuanto al grupo semitopológico en cuestión, solamente escribiremos $\mathcal{N}(e)$.

Proposición 1.2.5. Si $G$ es un grupo semitopológico, entonces para cualquier subconjunto $A$ de $G$ se satisface:

$$
\bar{A}=\bigcap_{U \in \mathcal{N}(e)} A U^{-1}=\bigcap_{U \in \mathcal{N}(e)} U^{-1} A .
$$

Demostración. El punto $x$ está en $\bar{A}$ si y sólo si la intersección $x U \cap A$ no es vacía para cada $U \in \mathcal{N}(e)$, equivalentemente, $x \in A U^{-1}$ para cada $U \in \mathcal{N}(e)$. Lo anterior muestra que $\bar{A}=\bigcap_{U \in \mathcal{N}(e)} A U^{-1}$. De forma similar se prueba que $\bar{A}=\bigcap_{U \in \mathcal{N}(e)} U^{-1} A$.

Corolario 1.2.6. Sea $A$ un subconjunto de un grupo semitopológico $G$. Entonces, $\bar{A} \subseteq A U^{-1}$ y $\bar{A} \subseteq U^{-1} A$ para cada $U \in \mathcal{N}(e)$.

Proposición 1.2.7. Sea $G$ un grupo paratopológico. Si $U, V \in \mathcal{N}(e)$ satisfacen $V^{2} \subseteq U$, entonces $\overline{V^{-1}} \subseteq U^{-1}$.

Demostración. Por el corolario anterior, $\overline{V^{-1}} \subseteq V^{-1} V^{-1} \subseteq U^{-1}$.

\subsection{Grupo topológico asociado}

Dado un grupo paratopológico podemos asignarle de forma funtorial un grupo topológico de la siguiente manera:

Sea $G$ un grupo paratopológico con topología $\tau$, definimos la topología conjugada $\tau^{-1}$ en $G$ como $\tau^{-1}=\left\{U^{-1}: U \in \tau\right\}$. Hagamos $G^{\prime}=\left(G, \tau^{-1}\right)$. Entonces $G^{\prime}$ es un grupo paratopológico homeomorfo a $G$. El supremo $\tau^{*}=$ $\tau \vee \tau^{-1}$ es una topología de grupo en $G$ y $G^{*}=\left(G, \tau^{*}\right)$ se llama el grupo topológico asociado a $G$. Observemos que $\tau^{*}$ es la mínima de las topologías de grupo en $G$ que contienen a $\tau$. 
El siguiente resultado describe la topología del grupo topológico asociado $G^{*}$ en términos de la topologia de $G$.

Proposición 1.3.1. Si $\mathcal{B}$ es una base local de la identidad en el grupo paratopológico $G$, entonces $\mathcal{B}^{*}=\left\{U \cap U^{-1}: U \in \mathcal{B}\right\}$ es una base local de la identidad en $G^{*}$. Además, si $G$ es $T_{0}$, entonces $G^{*}$ es completamente regular.

Demostración. La primera parte es inmediata de la definición de grupo topológico asociado. Supongamos que $G$ es $T_{0}$. Como la topología en $G^{*}$ es más fina que la topología en $G$, el grupo $G^{*}$ es $T_{0}$. Para finalizar la demostración basta recordar que en grupos topológicos ser $T_{0}$ es equivalente a ser completamente regular.

El siguiente resultado nos permite estudiar algunas propiedades topológicas del grupo topológico asociado $G^{*}$ en términos del grupo paratopológico G.

Proposición 1.3.2. ([5], [30]) Sea $G$ un grupo paratopológico. Si consideramos la diagonal $\Delta_{G}=\{(x, x): x \in G\}$ con la topología que hereda del producto $G \times G^{\prime}$, entonces $\Delta_{G}$ es un grupo topológico el cual es topológicamente isomorfo al grupo $G^{*}$. Además, si $G$ es $T_{1}$, el grupo $\Delta_{G}$ es cerrado en $G \times G^{\prime}$.

Demostración. Es claro que $\Delta_{G}$ es un grupo algebraico y por lo tanto, es un grupo paratopológico si se le considera con la topología heredada de $G \times G^{\prime}$. Sea $d: G^{*} \rightarrow \Delta_{G}$ la función definida como $f(x)=(x, x)$ para cada $x \in$ $G^{*}$. Como $G^{*}=\left(G, \tau \vee \tau^{-1}\right)$, la función $d$ es un homeomorfismo (ver [6, Proposition 1.6.1]). Es claro que $f$ es un isomorfismo de grupos. Se sigue de lo anterior que $\Delta_{G}$ es un grupo topológico el cual es topológicamente isomorfo al grupo $G^{*}$.

Por último, supongamos que $G$ es $T_{1}$. Tomemos $x, y \in G$ con $x \neq y$, entonces $x y^{-1} \neq e$. Como $G$ es $T_{1}$ podemos encontrar una vecindad $W$ de $x y^{-1}$ en $G$ tal que $e \notin W$. Por la continuidad de la multiplicación en $G$, existen vecindades $U, V$ de $x$ y $y^{-1}$, las cuales satisfacen $x y^{-1} \in U V \subseteq W$. En consecuencia $e \notin U V$. Por lo tanto, $U \times V^{-1}$ es una vecindad de $(x, y)$ en $G \times G^{\prime}$ la cual no intersecta a la diagonal $\Delta_{G}$.

Lema 1.3.3. ([30]) Sea $G$ un grupo paratopológico. Entonces se satisface lo siguiente:

a) Si $G$ es primero (segundo) numerable, entonces $G^{*}$ también lo es;

b) Si $G$ es $T_{1}$ y $\sigma$-compacto, $G^{*}$ es Tychonoff $\sigma$-compacto. 


\subsection{Regularización}

Dado un espacio $(X, \tau)$, se define la semirregularización de $\tau$ como $\tau_{r}=$ $\{$ int $\bar{U}: U \in \tau\}$. La topología $\tau_{r}$ es más débil que $\tau$ y el espacio $r(X)=$ $\left(X, \tau_{r}\right)$ es llamado la semirregularización de $X$. Es fácil probar que si $X$ es Hausdorff, el espacio $r(X)$ es Hausdorff y semirregular, es decir, la familia de abiertos regulares en $r(X)$ forma una base para $r(X)$. Si $(G, \tau)$ es un grupo paratopológico, entonces al espacio $r(G)=\left(G, \tau_{r}\right)$ lo llamaremos la regularización de $G$ debido al siguiente hecho:

Teorema 1.4.1. ([26, Example 1.9]) Si $(G, \tau)$ es un grupo paratopológico Hausdorff, entonces $r(G)=\left(G, \tau_{r}\right)$ es un grupo paratopológico regular.

Demostración. La familia $\mathcal{B}_{r}=\left\{i n t \bar{U}: U \in \mathcal{N}_{G}(e)\right\}$ es una base local de la identidad $e$ en $r(G)$. Veamos que $r(G)$ es un grupo paratopológico; para ello, es suficiente probar que la familia $\mathcal{B}_{r}$ satisface las condiciones de Pontryagin que aparecen en el teorema 1.2.1.

La parte i) se cumple porque la intersección de dos abiertos regulares es un abierto regular.

Para la condición ii), tomemos $U \in \mathcal{N}_{G}(e)$ y $x \in$ int $\bar{U}$. Como int $\bar{U}$ es abierto en $G$, existe $V \in \mathcal{N}_{G}(e)$ tal que $V x \subseteq i n t \bar{U}$. En consecuencia, $(\operatorname{int} \bar{V}) x=\operatorname{int}(\overline{V x}) \subseteq \operatorname{int}(\overline{i n t \bar{U}})=\operatorname{int} \bar{U}$.

La parte iii) se satisface debido a que las traslaciones izquierdas y derechas son homeomorfismos de $G$ en $G$.

Probemos la condición iv). Tomemos $U \in \mathcal{N}_{G}(e)$. Como $G$ es un grupo paratopológico, existe $V \in \mathcal{N}_{G}(e)$ tal que $V^{2} \subseteq U$. Por la continuidad de la multiplicación en $G$, tenemos que $\bar{V}^{2} \subseteq \bar{U}$. Como int $\bar{V}$ es abierto en $G$, tenemos que $(\operatorname{int} \bar{V})^{2} \subseteq \operatorname{int}\left(\bar{V}^{2}\right) \subseteq \operatorname{int} \bar{U}$.

De lo anterior podemos concluir que $r(G)$ es un grupo paratopológico. Como $G$ es Hausdorff, el espacio $r(G)$ es Hausdorff. Veamos que $r(G)$ es regular. La semirregularización de un espacio $G$ tiene los mismos abiertos regulares que el espacio $G$. Sabemos que la familia $\mathcal{B}_{r}$ consiste de abiertos regulares en $G$, por lo tanto, la familia $\mathcal{B}_{r}$ está formada de abiertos regulares en $r(G)$. Tomemos $O \in \mathcal{B}_{r}$. La igualdad $i n t_{\tau_{r}} \bar{O}^{\tau_{r}}=O$ se satisface porque $O$ es un abierto regular en $r(G)$. Como $r(G)$ es un grupo paratopológico, existe $W \in \mathcal{B}_{r}$ tal que $W^{2} \subseteq O$. En consecuencia $W \bar{W}^{\tau_{r}} \subseteq \bar{W}^{\tau_{r}} \bar{W}^{\tau_{r}} \subseteq \bar{O}^{\tau_{r}}$. Así, $\bar{W}^{\tau_{r}} \subseteq i n t_{\tau_{r}} \bar{O}^{\tau_{r}}=O$. Hemos probado que $r(G)$ es un grupo paratopológico regular. 


\section{Capítulo 2}

\section{Encajes}

En este capítulo caracterizamos internamente a los subgrupos de productos de grupos paratopológicos $T_{i}$ primero (segundo) numerables, para $i=0,1$ (ver teoremas 2.2.8, 2.2.10, 2.2.11 y 2.2.14). Para ello necesitamos el concepto de número de simetría. Además, el ejemplo 2.2.22 responde algunas preguntas planteadas por M. Sanchis y M. Tkachenko en [30] y [32].

\subsection{Número de simetría}

Recordemos que $\mathcal{N}(e)$ denota al conjunto de vecindades abiertas de $e$ en $G$ (ver definición 1.2.4). Un grupo semitopológico $G$ es $T_{1}$ si y sólo si para cada $x \in G \backslash\{e\}$, existe $V \in \mathcal{N}(e)$ tal que $x \notin V$, es decir, $\bigcap_{V \in \mathcal{N}(e)} V=\{e\}$, equivalentemente, $\bigcap_{V \in \mathcal{N}(e)} V^{-1}=\{e\}$. Con base en esto tenemos la siguiente definición.

Definición 2.1.1. Sea $G$ un grupo semitopológico $T_{1}$. El número de simetría de $G$, denotado por $\operatorname{Sm}(G)$, es el mínimo cardinal $\kappa$ tal que para cada vecindad $U$ de e en $G$, existe una familia $\gamma \subseteq \mathcal{N}(e)$ tal que $\bigcap_{V \in \gamma} V^{-1} \subseteq U y$ $|\gamma| \leq \kappa$

Un grupo semitopológico $T_{1} G$ satisface $\operatorname{Sm}(G)=1$ si y sólo si $G$ es un grupo cuasitopológico, es decir, un grupo semitopológico con inversa continua. Las siguientes tres proposiciones se deducen fácilmente de la definición de número de simetría (los espacios involucrados en dichas proposiciones son considerados $\left.T_{1}\right)$. 
Proposición 2.1.2. Si $K$ es un subgrupo del grupo semitopológico $G$, entonces se tiene la desigualdad $\operatorname{Sm}(K) \leq \operatorname{Sm}(G)$.

Proposición 2.1.3. Cada grupo semitopológico $G$ satisface $\operatorname{Sm}(G) \leq \psi(G)$, donde $\psi(G)$ es el pseudocarácter de $G$.

Proposición 2.1.4. Sea $G=\prod_{i \in I} G_{i}$ el producto de una familia de grupos semitopológicos tales que $\operatorname{Sm}\left(G_{i}\right) \leq \kappa$ para cada $i \in I$. Entonces $\operatorname{Sm}(G) \leq \kappa$.

El siguiente resultado relaciona el número de Lindelöf y el número de simetría de un grupo paratopológico $G$.

Proposición 2.1.5. Si $G$ es un grupo paratopológico $T_{1}$, entonces se satisface la desigualdad $\operatorname{Sm}(G) \leq l(G)$.

Demostración. Tomemos $U \in \mathcal{N}(e)$. Como $G$ es un grupo paratopológico $T_{1}$, para cada $x \in G \backslash U$ existe $V_{x} \in \mathcal{N}(e)$ tal que $e \notin x V_{x}^{2}$, es decir, $V_{x}^{-1} \cap x V_{x}=\emptyset$. La familia $\left\{x V_{x}: x \in G \backslash U\right\}$ es una cubierta abierta del cerrado $G \backslash U$, por lo tanto, existe un subconjunto $S \subseteq G \backslash U$ tal que $|S| \leq l(G)$ y la familia de abiertos $\left\{x V_{x}: x \in S\right\}$ cubre a $G \backslash U$. De lo anterior se sigue que $\bigcap_{x \in S} V_{x}^{-1} \subseteq U$ y $|S| \leq l(G)$. Por lo tanto, $\operatorname{Sm}(G) \leq l(G)$.

En un intento de extender la proposición anterior a grupos semitopológicos se plantea el siguiente problema.

Problema 2.1.6. ¿Cada grupo semitopológico regular $G$ cumple la desigual$\operatorname{dad} \operatorname{Sm}(G) \leq l(G)$ ?

Un espacio $X$ es un $P$-espacio si cada conjunto $G_{\delta}$ en $X$ es abierto. Sea $(G, \tau)$ un grupo semitopológico (paratopológico). La familia de conjuntos $G_{\delta}$ en $(G, \tau)$ forma una base para una topología $\tau_{\omega}$ en $G$. Esta topología es llamada la $G_{\delta}$-modificación de $\tau$. Es fácil probar que $(G)_{\omega}=\left(G, \tau_{\omega}\right)$ es un grupo semitopológico (paratopológico). Es claro que $(G)_{\omega}$ es un $P$-espacio. Más aún, $G$ es un $P$-espacio si y sólo si $G=(G)_{\omega}$. El siguiente hecho es auxiliar en la prueba de la proposición 2.1.8.

Proposición 2.1.7. Sean $G$ un grupo semitopológico y $(G)_{\omega}$ su $G_{\delta}$-modificación. Entonces $(G)_{\omega}$ es un grupo cuasitopológico si y sólo si $\operatorname{Sm}(G) \leq \omega$. 
Demostración. Denotemos por $\mathcal{N}_{\omega}(e)$ al conjunto de vecindades de la identidad en $(G)_{\omega}$. Supongamos que $(G)_{\omega}$ es un grupo cuasitopológico. Tomemos $U \in \mathcal{N}(e)$, entonces $U$ está en $\mathcal{N}_{\omega}(e)$. Por hipótesis, existe $V \in \mathcal{N}_{\omega}(e)$ tal que $V^{-1} \subseteq U$. Podemos encontrar una familia $\left\{V_{n}: n \in \omega\right\} \subseteq \mathcal{N}(e)$ tal que $\bigcap_{n \in \omega} V_{n} \subseteq V$, por lo tanto, $\bigcap_{n \in \omega} V_{n}^{-1} \subseteq U$, lo cual implica que $\operatorname{Sm}(G) \leq \omega$. Recíprocamente, supongamos que $\operatorname{Sm}(G) \leq \omega$. Veamos que $(G)_{\omega}$ es un grupo cuasitopológico. Sea $U \in \mathcal{N}_{\omega}(e)$, entonces existe una familia $\left\{U_{n}: n \in \omega\right\} \subseteq \mathcal{N}(e)$ tal que $\bigcap_{n \in \omega} U_{n} \subseteq U$. Por hipótesis, para cada $n \in \omega$, existe una familia numerable $\gamma_{n} \subseteq \mathcal{N}(e)$ tal que $\bigcap_{W \in \gamma_{n}} W^{-1} \subseteq U_{n}$. Sea $\gamma=\bigcup_{n \in \omega} \gamma_{n}$, es claro que $V=\bigcap_{W \in \gamma} W$ es un conjunto que satisface $V \in \mathcal{N}_{\omega}(e)$ y $V^{-1} \subseteq U$.

En [30], M. Sanchis y M. Tkachenko demuestran que si $H$ es un grupo paratopológico Hausdorff tal que $H$ es un $P$-espacio Lindelöf, entonces $H$ es un grupo topológico. La siguiente proposición muestra que el resultado es válido si debilitamos la condición de espacio Hausdorff a $T_{1}$.

Proposición 2.1.8. Si $G$ es un grupo paratopológico $T_{1}$ tal que $G$ es un $P$-espacio Lindelöf, entonces $G$ es un grupo topológico.

Demostración. Consideremos $G$ como en las hipótesis. Por la proposición 2.1.5 tenemos $\operatorname{Sm}(G) \leq \omega$. Como $G=G_{\omega}$ por ser $G$ un $P$-espacio, aplicando la proposición 2.1.7 obtenemos el resultado.

Sean $X$ un espacio y $A \subseteq X$, definimos la $G_{\delta}$-cerradura de $A$ en $X$ como el conjunto de puntos $x \in X$ tales que cada conjunto $G_{\delta}$ en $X$ que contiene a $x$ intersecta a $A$. Dado un grupo paratopológico $G$ y un subgrupo $H$ de $G$, en general la $G_{\delta}$-cerradura de $H$ no es un subgrupo de $G$ como se muestra a continuación.

Ejemplo 2.1.9. Consideremos el conjunto de enteros $\mathbb{Z}$ y $\kappa$ un cardinal no numerable. Para cada subconjunto finito $A \subseteq \kappa$, definamos el conjunto $U_{A} \subseteq \mathbb{Z}^{\kappa}$ como

$$
U_{A}=\left\{x \in \mathbb{Z}^{\kappa}: x(\alpha)=0 \text { si } \alpha \in A \text { y } x(\alpha) \geq 0 \text { si } \alpha \in \kappa \backslash A\right\} .
$$

Existe una topología $\tau$ en $\mathbb{Z}^{\kappa}$ tal que $G=\left(\mathbb{Z}^{\kappa}, \tau\right)$ es un grupo paratopológico completamente regular y la familia $\mathcal{U}=\left\{U_{A}: A \subseteq \kappa,|A|<\omega\right\}$ es una base local de la identidad en $\mathbb{Z}^{\kappa}$ (ver [32, Example 2.9]). Sea $H=\{x \in$ $G:|\operatorname{supp}(x)| \leq \omega\}$, donde $\operatorname{supp}(x)=\{\alpha \in \kappa: x(\alpha) \neq 0\}$. Denotemos

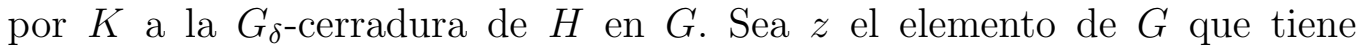


todas sus coordenadas igual a -1 . Veamos que $z \in K$. Tomemos una familia $\gamma=\left\{U_{A(n)}: n \in \omega\right\}$ de vecindades abiertas de la identidad en $G$. El conjunto $U=\bigcap \gamma$ es un conjunto $G_{\delta}$ en $G$. Hagamos $F=\bigcup_{n \in \omega} A(n)$. Observemos que $F$ es un conjunto numerable. Además, se satisface la igualdad

$$
U=\left\{x \in \mathbb{Z}^{\kappa}: x(\alpha)=0 \text { si } \alpha \in F \text { y } x(\alpha) \geq 0 \text { si } \alpha \in \kappa \backslash F\right\} .
$$

Definamos $y \in \mathbb{Z}^{\kappa}$ de la siguiente manera: $y(\alpha)=-1$ si $\alpha \in F$ y $y(\alpha)=0$ si $\alpha \in \kappa \backslash F$. Es claro que $y \in H \cap(z+U)$. De lo anterior podemos concluir que $z \in K$. Hagamos $V=U_{\emptyset}$. Tenemos que $H \cap(-z+V)=\emptyset$, en consecuencia $-z \notin K$. Concluimos que $K$ no es subgrupo de $G$.

En el siguiente resultado damos condiciones suficientes para que la $G_{\delta^{-}}$ cerradura de un subgrupo en un grupo semitopológico también sea un subgrupo.

Proposición 2.1.10. Sea $G$ un grupo semitopológico con $S m(G) \leq \omega$. Si $H$ es un subgrupo de $G$, entonces la $G_{\delta}$-cerradura de $H$ en $G$ también es un subgrupo de $G$.

Demostración. Consideremos a $G$ como en las hipótesis. Sea $H$ un subgrupo de $G$. Como $\operatorname{Sm}(G) \leq \omega$, el grupo $(G)_{\omega}$ es cuasitopológico por la proposición 2.1.7. La cerradura de un subgrupo de un grupo cuasitopológico es un subgrupo (ver [6, Proposition 1.4.13]). Es claro que la cerradura de $H$ en $(G)_{\omega}$ es un subgrupo y coincide con la $G_{\delta}$-cerradura de $H$ en $G$.

Un grupo paratopológico $G$ es 2-pseudocompacto si $\bigcap_{n \in \omega} \overline{U_{n}^{-1}} \neq \emptyset$ para cada sucesión decreciente $\left\{U_{n}: n \in \omega\right\}$ de abiertos no vacíos en $G$. En 2007 O. Alas y M. Sanchis plantean en [1] la siguiente cuestión: ¿es cada grupo paratopológico 2-pseudocompacto de pseudocarácter numerable un grupo topológico? Ravsky en [24] responde en forma positiva a esta pregunta. Todo grupo paratopológico de pseudocarácter numerable tiene número de simetría numerable (ver proposición 2.1.3) pero no recíprocamente. Por ejemplo, sea $\mathbb{S}$ la recta de Sorgenfrey y $\kappa$ un cardinal no numerable, entonces por las proposiciones 2.1.3 y 2.1.4 se tiene que $S m\left(\mathbb{S}^{\kappa}\right) \leq \omega$. Sin embargo, el pseudocarácter de $\mathbb{S}^{\kappa}$ no es numerable. De esta observación se sigue que la siguiente caracterización generaliza el resultado de Ravsky.

Proposición 2.1.11. Sea $G$ un grupo paratopológico 2-pseudocompacto. Entonces $G$ es un grupo topológico si y sólo si $\operatorname{Sm}(G) \leq \omega$. 
Demostración. Es claro que si $G$ es un grupo topológico, entonces $\operatorname{Sm}(G) \leq$ $\omega$. Probemos el recíproco. Supongamos que $G$ tiene número de simetría numerable. Sea $U \in \mathcal{N}(e)$. Por la continuidad de la multiplicación en $G$ existe $V \in$ $\mathcal{N}(e)$ tal que $V^{2} \subseteq U$. Por hipótesis, existe una sucesión $\left\{V_{n}: n \in \omega\right\} \subseteq \mathcal{N}(e)$ tal que $\bigcap V_{n}^{-1} \subseteq V$. Como $G$ es un grupo paratopológico, podemos suponer que $V_{n+1}^{2} \subseteq V_{n}$ para cada $n \in \omega$. Aplicando la proposición 1.2.7, tenemos que $\overline{V_{n+1}^{-1}} \subseteq V_{n}^{-1}$ para todo $n \in \omega$ y, por lo tanto, $\bigcap_{n \in \omega} \overline{V_{n}^{-1}}=\bigcap_{n \in \omega} V_{n}^{-1}$. Si existe $n \in \omega$ tal que $V_{n} \subseteq \overline{V^{-1}}$, entonces $V_{n} \subseteq \overline{V^{-1}} \subseteq V^{-2} \subseteq U^{-1}$ con lo cual terminaríamos la demostración. Supongamos lo contrario, es decir, $V_{n} \backslash \overline{V^{-1}} \neq \emptyset$ para cada $n \in \omega$. Tenemos lo siguiente:

$$
\bigcap_{n \in \omega} \overline{V_{n}^{-1} \backslash\left(\overline{V^{-1}}\right)^{-1}} \subseteq \bigcap_{n \in \omega} \overline{V_{n}^{-1} \backslash V} \subseteq\left(\bigcap_{n \in \omega} \overline{V_{n}^{-1}}\right) \backslash V=\left(\bigcap_{n \in \omega} V_{n}^{-1}\right) \backslash V=\emptyset .
$$

Lo anterior contradice la 2-pseudocompacidad de $G$. Por lo tanto, $G$ es un grupo topológico.

Corolario 2.1.12. Sea $G=\prod_{i \in I} G_{i}$ el producto de grupos paratopológicos Lindelöf. Si $K$ es un subgrupo 2-pseudocompacto de $G$, entonces $K$ es un grupo topológico.

Demostración. Por las proposiciones 2.1.2, 2.1.4 y 2.1.5 se tiene que $\operatorname{Sm}(K) \leq$ $\omega$. Como $K$ es 2-pseudocompacto, aplicando la proposición 2.1.11 obtenemos el resultado.

El siguiente lema es útil en la demostración de la proposición 2.1.14.

Lema 2.1.13. Sea $G$ un grupo paratopológico Hausdorff el cual es un Pespacio. Entonces la regularización $r(G)$ de $G$ también es un P-espacio.

Demostración. Consideremos una sucesión $\left\{U_{n}: n \in \omega\right\}$ de conjuntos abiertos en $r(G)$. Tomemos $x \in \bigcap_{n \in \omega} U_{n}$. Como $r(G)$ es un espacio regular (ver teorema 1.4.1), existe $V_{n} \in \tau_{r}$ tal que $x \in V_{n} \subset \bar{V}_{n}^{\tau_{r}} \subset U_{n}$, para cada $n \in \omega$. Como $\tau_{r} \subseteq \tau, x \in V_{n} \subseteq \bar{V}_{n}^{\tau} \subseteq \bar{V}_{n}^{\tau_{r}} \subseteq U_{n}$, para todo $n$. Sea $V=\bigcap_{n \in \omega} V_{n}$. Tenemos lo siguiente

$$
x \in V \subseteq \operatorname{Int}_{\tau} \bar{V}^{\tau} \subseteq \bar{V}^{\tau} \subseteq \bigcap_{n \in \omega} \bar{V}_{n}^{\tau} \subseteq \bigcap_{n \in \omega} U_{n} .
$$

Esto termina la demostración. 
Un espacio $X$ se dice débilmente compacto si cada familia localmente finita de abiertos en $X$ es finita. En la clase de espacios Tychonoff, compacidad débil es equivalente a pseudocompacidad.

Es bien sabido que todo $P$-espacio pseudocompacto es finito. En grupos paratopológicos débilmente compactos Hausdorff sucede algo similar.

Proposición 2.1.14. Sea $G$ un grupo paratopológico débilmente compacto Hausdorff tal que $G$ es un P-espacio. Entonces $G$ es finito.

Demostración. Sea $G$ como en las hipótesis. Como la topología en $r(G)$ es más débil que la topología en $G$ y por el teorema 1.4.1, su regularización $r(G)$ es un grupo paratopológico débilmente compacto regular. Todo grupo paratopológico débilmente compacto regular es un grupo topológico pseudocompacto (ver [5, Theorem 1.7]). Por lo tanto, $r(G)$ es un grupo paratopológico pseudocompacto. Aplicando el lema 2.1.13, tenemos que $r(G)$ es un $P$-espacio. Concluimos que $G$ es finito.

Dado un grupo $G$, un subconjunto $S \subseteq G$ es un subsemigrupo de $G$ si para cada $x, y \in S$ se tiene que $x y \in S$. Sean $G$ un grupo abeliano y $S$ un subsemigrupo de $G$; entonces existe una topología $\tau_{S}^{*}$ en $G$ tal que $G_{S}^{*}=\left(G, \tau_{S}^{*}\right)$ es un grupo paratopológico y la familia de conjuntos $\mathcal{B}_{S}=$ $\{\{0\} \cup(x+S): x \in S\}$ es una base local de la identidad. La topología $\tau_{S}^{*}$ es llamada la topología cono* generada por el subsemigrupo $S$ en $G$ (ver [24]).

El siguiente ejemplo muestra que en la proposición anterior, la condición de ser un espacio Hausdorff no puede ser sustituida por $T_{1}$.

Ejemplo 2.1.15. Existe un P-grupo paratopológico débilmente compacto $T_{1}$ de cardinalidad infinita.

Sea $G=\bigoplus_{\alpha \in \omega_{1}} \mathbb{Z}$, es decir, la suma directa de $\omega_{1}$ copias del grupo de los enteros $\mathbb{Z}$. Definamos $S$ como sigue:

$S=\left\{0_{G}\right\} \cup\left\{x \in G:\left(\exists \beta \in \omega_{1}\right)((\forall \alpha>\beta)(x(\alpha)=0)\right.$ y $\left.(x(\beta)>0))\right\}$.

El grupo paratopológico $G_{S}^{*}$ es débilmente compacto $T_{1}$ (ver [24]). Es claro que el grupo $G_{S}^{*}$ es infinito. Veamos que $G_{S}^{*}$ es un $P$-espacio. Para ello, es suficiente probar que para cada familia numerable $\gamma=\left\{U_{n}: n \in \omega\right\}$ de vecindades abiertas del $0_{G}$ en $G_{S}^{*}$, se tiene $0_{G} \in \operatorname{Int}(\bigcap \gamma)$. Por la definición de la topología cono*, podemos encontrar una sucesión $\left\{x_{n}: n \in \omega\right\} \subseteq S$ tal que $V_{n}=\left\{0_{G}\right\} \cup\left(x_{n}+S\right) \subseteq U_{n}$ para cada $n \in \omega$. También, existe una suceción $\left\{\beta_{n}: n \in \omega\right\} \subseteq \omega_{1}$ tal que para cada $n \in \omega$ se cumple $x_{n}(\alpha)=0$ si $\alpha>\beta_{n}$ y $x_{n}\left(\beta_{n}\right)>0$. Como $\omega_{1}$ no es numerable, podemos encontrar $\beta \in \omega_{1}$ 
tal que $\beta>\beta_{n}$ para cada $n \in \omega$. Tomemos $x \in S$ el cual satisface $x(\alpha)=0$ si $\alpha \in \omega_{1} \backslash\{\beta\}$ y $x(\beta)=1$. Hagamos $V=\left\{0_{G}\right\} \cup(x+S)$. Es claro que $x-x_{n} \in S$, en consecuencia $x-x_{n}+S \subseteq S$, es decir, $x+S \subseteq x_{n}+S$ y, por lo tanto, $V \subseteq V_{n}$ para cada $n \in \omega$. Se sigue de lo anterior que $0_{G} \in V \subseteq$ $\bigcap_{n \in \omega} V_{n} \subseteq \bigcap_{n \in \omega} U_{n}$.

\section{2. $\quad$ El caso $T_{0}$ y $T_{1}$}

De acuerdo con [32], dada una propiedad topológica y/o algebraica $\mathcal{P}$, decimos que un grupo paratopológico $G$ es proyectivamente $\mathcal{P}$ si para cada $U \in \mathcal{N}_{G}\left(e_{G}\right)$, existe un homomorfismo continuo y sobreyectivo $p$ de $G$ en un grupo paratopológico $H$ con la propiedad $\mathcal{P}$ tal que $p^{-1}(V) \subseteq U$, para alguna $V \in \mathcal{N}_{G}\left(e_{H}\right)$. En el siguiente resultado damos una caracterización de los grupos paratopológicos $T_{0}$ que son proyectivamente $\mathcal{P}$, para alguna propiedad $\mathcal{P}$.

Proposición 2.2.1. Sean $G$ un grupo paratopológico $T_{0}$ y $\mathcal{P}$ una propiedad invariante bajo subgrupos y bajo productos finitos en la clase grupos paratopológicos. Entonces, $G$ es proyectivamente $\mathcal{P}$ si y sólo si $G$ es topológicamente isomorfo a un subgrupo de productos de grupos paratopológicos con la propiedad $\mathcal{P}$.

Demostración. Supongamos que $G$ es un grupo paratopológico proyectivamente $\mathcal{P}$. Sea $\left\{U_{i}: i \in I\right\}$ una base local de la identidad $e_{G}$ en $G$. Por hipótesis, para cada $U_{i}$, existe un homomorfismo continuo y sobreyectivo $p_{i}$ de $G$ en un grupo paratopológico $H_{i}$ con la propiedad $\mathcal{P}$ tal que $p^{-1}(V) \subseteq U_{i}$, para alguna vecindad $V$ de la identidad $e_{i}$ en $H_{i}$. Sean $p$ el producto diagonal de la familia de funciones $\left\{p_{i}: i \in I\right\}$ y $H=\prod_{i \in I} H_{i}$. Es claro que $p: G \rightarrow H$ es un homomosfismo continuo. Veamos que la familia $\left\{p_{i}: i \in I\right\}$ separa puntos y cerrados. Sea $x \in G$ y $F$ un cerrado en $G$ que no contiene a $x$. Como la familia $\left\{p_{i}: i \in I\right\}$ consta de homomorfismos continuos, podemos suponer que $x=e_{G}$. Tomemos $i \in I$ tal que $U_{i} \cap F=\emptyset$. Consideremos $p_{i}: G \rightarrow H_{i}$. Sabemos que existe una vecindad $V$ de la identidad $e_{i}$ en $H_{i}$ tal que $p^{-1}(V) \subseteq U_{i}$. Por lo tanto, $V \cap \overline{p_{i}(F)}=\emptyset$. En consecuencia, $e_{i} \notin \overline{p_{i}(F)}$. Hemos probado que la familia $\left\{p_{i}: i \in I\right\}$ separa puntos y cerrados. Además, como $G$ es $T_{0}$, también separa puntos. Por lo tanto, $G$ es topológicamente isomorfo al grupo paratopológico $p(G) \leq H$. 
Recíprocamente, supongamos que $G$ es topológicamente isomorfo a un subgrupo del producto $F=\prod_{i \in I} F_{i}$, donde cada $F_{i}$ es un grupo paratopológico con la propiedad $\mathcal{P}$. Existe un homomorfismo continuo e inyectivo $f: G \rightarrow F$ tal que $f: G \rightarrow f(G)$ es un homeomorfismo. Sea $U$ una vecindad abierta de en $G$. Existe un abierto canónico $W$ en $F$ que contiene a la identidad de $F$ tal que $W \cap f(G) \subseteq f(U)$. Hagamos $S=\operatorname{supp}(W)$. Sea $\pi_{S}$ la proyección de $F$ sobre $\prod_{s \in S} F_{s}$. El conjunto $\pi_{S}(W)$ es abierto en $\prod_{s \in S} F_{s}$. Sean $p=\pi_{S} \circ f$ y $H=p(G)$. Observemos que $H$ tiene la propiedad $\mathcal{P}$. Sea $V=\pi_{S}(W) \cap p(G)$. Se sigue de lo anterior que $p: G \rightarrow H$ es un homomorfismo continuo y sobreyectivo. Además, $V$ es una vecindad de la identidad en $H$ la cual satisface $\left.p^{-1}(V)=f^{-1}\left(\pi_{S}^{-1}(V)\right)=f^{-1}\left(\pi_{S}^{-1} \pi_{S}(W) \cap f(G)\right)\right) \subseteq$ $f^{-1}(W \cap f(G)) \subseteq U$.

A continuación caracterizamos a los grupos paratopológicos proyectivamente $T_{i}$ primero (segundo) numerables, para $i=0,1,2,3,3 \frac{1}{2}$.

Corolario 2.2.2. Sea $G$ un grupo paratopológico $T_{i}$. Entonces, $G$ es proyectivamente $T_{i}$ primero (segundo) numerable si y sólo si es topológicamente isomorfo a un subgrupo de un producto de grupos paratopológicos $T_{i}$ primero (segundo) numerables, para $i=0,1,2,3,3 \frac{1}{2}$.

Los siguientes lemas y definiciones nos servirán para dar una caracterización interna de los grupos paratopológicos que son topológicamente isomorfos a un subgrupo de un producto de grupos paratopológicos $T_{i}$ primero (segundo) numerables para $i=0,1$.

Un subconjunto $V$ de un grupo semitopológico $G$ se llama $\omega$-bueno si existe una familia numerable $\gamma \subset \mathcal{N}_{G}(e)$ tal que para cada $x \in V$ podemos encontrar $W \in \gamma$ con $x W \subseteq V$.

Lema 2.2.3. ([30, Lemma 3.10]) Cada grupo paratopológico G tiene una base local en la identidad la cual consiste de conjuntos $\omega$-buenos.

Definición 2.2.4. Sea $G$ un grupo semitopológico con identidad e. Decimos que una familia $\gamma \subseteq \mathcal{N}(e)$ está subordinada a una vecindad $U$ de e, si para cada $x \in G$, existe $V \in \gamma$ tal que $x V x^{-1} \subseteq U$.

Definición 2.2.5. Un grupo semitopológico $G$ se llama $\omega$-balanceado si para cada $U \in \mathcal{N}(e)$, existe una familia numerable $\gamma \subseteq \mathcal{N}(e)$ subordinada a $U$. 
Lema 2.2.6. Sea $G$ un grupo paratopológico. Supongamos que una familia $\gamma \subset \mathcal{N}(e)$ satisface las siguientes condiciones:

(a) para cada $U \in \gamma$, existe $V \in \gamma$ tal que $V^{2} \subset U$;

(b) $\gamma$ está subordinada a $U$ para cada $U \in \gamma$.

Entonces el conjunto $N=\bigcap\left\{U \cap U^{-1}: U \in \gamma\right\}$ es un subgrupo invariante de G. Si además, se cumple:

(c) $\bigcap\left\{V^{-1}: V \in \gamma\right\} \subseteq U$ para cada $U \in \gamma$, entonces $N=\bigcap\{U: U \in \gamma\}$ y el subgrupo $N$ es cerrado en $G$.

Demostración. Supongamos que se satisfacen (a) y (b). Es claro que $N=$ $N^{-1}$. Sean $a, b \in N$ y $U \in \gamma$. Tomemos $V \in \gamma$ tal que $V^{2} \subset U$, entonces $a, b \in V \cap V^{-1}$. Se sigue que $a b \in V^{2} \subseteq U$ y $a b \in V^{-2} \subseteq U^{-1}$. Por lo tanto, $a b \in U \cap U^{-1}$. Lo anterior muestra que $N$ es un subgrupo de $G$. Ahora, veamos que $N$ es invariante en $G$. Sean $h \in H, x \in G$ y $U \in \gamma$. Por (b), podemos encontrar $V \in \gamma$ tal que $x V x^{-1} \subseteq U$. Como $h \in N$, entonces $h \in V \cap V^{-1}$. Por lo tanto, $x h x^{-1} \in x V x^{-1} \subseteq U$ y $x h x^{-1} \in x V^{-1} x^{-1} \subseteq U^{-1}$, es decir, $x h x^{-1} \in U \cap U^{-1}$.

Si suponemos que además de (a) y (b) se cumple (c), entonces $\bigcap_{U \in \gamma} U^{-1} \subseteq$ $\bigcap_{U \in \gamma} U$ y $\bigcap_{U \in \gamma} U \subseteq \bigcap_{U \in \gamma} U^{-1}$. Se sigue de lo anterior que $N=\bigcap_{U \in \gamma} U$. Por último, probemos que $N$ es cerrado. Sea $x \in G \backslash N$. Escojamos $U \in \gamma$ tal que $x \notin U^{-1}$ y tomemos $V \in \gamma$ tal que $V^{2} \subset U$. Afirmamos que $x V \cap N=\emptyset$, de lo contrario $x \in N V^{-1} \subseteq V^{-2} \subseteq U^{-1}$. Esto último es una contradicción.

Definición 2.2.7. Un grupo semitopológico $G$ es w-estrecho si para cada $U \in \mathcal{N}(e)$, existe un conjunto numerable $F \subseteq G$ tal que $G=F U=U F$.

Katz demuestra que un grupo topológico $G$ es topológicamente isomorfo a un subgrupo de un producto de grupos topológicos primero numerables (metrizables) si y sólo si es $\omega$-balanceado. Guran prueba que un grupo topológico $G$ es topológicamente isomorfo a un subgrupo de un producto de grupos topológicos segundo numerables si y sólo si es $\omega$-estrecho. Es bien conocido que en grupos topológicos ser $T_{0}$ es equivalente a ser completamente regular. Sin embargo, en grupos paratopológicos esto no sucede. Por esta razón, las versiones de los teoremas de Katz y Guran en grupos paratopológicos tienen que dividirse en casos, de acuerdo a los axiomas de separación. En [32], Tkachenko presenta las versiones de los teoremas de Katz y Guran para grupos paratopológicos Hausdorff y regulares. A continuación presentamos los casos para grupos paratopológicos $T_{0}$ y $T_{1}$. 
Teorema 2.2.8. Si $G$ un grupo paratopológico $T_{0}$, entonces $G$ es topológicamente isomorfo a un subgrupo de un producto de grupos paratopológicos $T_{0}$ primero numerables si y sólo si es $\omega$-balanceado.

Demostración. Supongamos que $G$ es un subgrupo de $\Pi=\prod_{i \in I} H_{i}$ donde $H_{i}$ es un grupo paratopológico $T_{0}$ primero numerable para cada $i \in I$. Como cada $H_{i}$ es primero numerable, cada $H_{i}$ es $\omega$-balanceado y, por lo tanto, el producto $\Pi$ es $\omega$-balanceado. Como la propiedad de ser $\omega$-balanceado se hereda a subgrupos, podemos concluir que $G$ es $\omega$-balanceado.

Ahora supongamos que $G$ es un grupo paratopológico $\omega$-balanceado $T_{0}$. Sea $U_{0} \in \mathcal{N}_{G}(e)$. Debemos definir un homomorfismo continuo sobreyectivo $p: G \rightarrow H$, donde $H$ es un grupo paratopológico primero numerable $T_{0} \mathrm{y}$ tenemos que encontrar una vecindad abierta $V_{0}$ de la identidad en $H$ tal que $p^{-1}\left(V_{0}\right) \subseteq U_{0}$.

Denotemos por $\mathcal{N}^{*}(e)$ la subfamilia de $\mathcal{N}_{G}(e)$ la cual consiste de conjuntos $\omega$-buenos. Por el lema 2.2.3, el conjunto $\mathcal{N}^{*}(e)$ es una base local de $e$ en $G$. Definamos inductivamente una sucesión $\left\{\gamma_{n}: n \in \omega\right\}$ de la siguiente forma.

Sea $U_{0}^{*} \in \mathcal{N}^{*}(e)$ tal que $U_{0}^{*} \subseteq U_{0}$. Hagamos $\gamma_{0}=\left\{U_{0}^{*}\right\}$. Supongamos que para algún $n \in \omega$ hemos definido $\gamma_{0}, \ldots, \gamma_{n}$ de tal forma que para cada $k \leq n$ se satisface:

(i) $\gamma_{k} \subseteq \mathcal{N}^{*}(e)$ y $\left|\gamma_{k}\right| \leq \omega$;

(ii) $\gamma_{k-1} \subseteq \gamma_{k}$;

(iii) $\gamma_{k}$ es cerrada bajo intersecciones finitas;

(iv) para cada $U \in \gamma_{k-1}$, existe $V \in \gamma_{k}$ tal que $V^{2} \subseteq U$;

(v) la familia $\gamma_{k}$ está subordinada a $U$, para cada $U \in \gamma_{k-1}$;

(vi) para cada $U \in \gamma_{k-1}$ y $x \in U$, existe $V \in \gamma_{k}$ tal que $x V \subseteq U$.

Como $\gamma_{n}$ es numerable, existe una familia numerable $\lambda_{n, 1} \subseteq \mathcal{N}^{*}(e)$ tal que cada $U \in \gamma_{n}$ contiene el cuadrado de algún elemento $V \in \lambda_{n, 1}$. Por ser $G$ w-balanceado, podemos encontrar una familia numerable $\lambda_{n, 2} \subseteq \mathcal{N}^{*}(e)$ subordinada a cada $U \in \gamma_{n}$. Como $\gamma_{n} \subseteq \mathcal{N}^{*}(e)$, existe una familia numerable $\lambda_{n, 3} \subseteq \mathcal{N}^{*}(e)$ tal que para cada $U \in \gamma_{n}$ y $x \in U$, existe $V \in \lambda_{n, 3}$ la cual satisface $x V \subseteq U$. Sea $\gamma_{n+1}$ la mínima familia que contiene a $\gamma_{n} \cup \bigcup_{i=1}^{3} \lambda_{n, i}$ y es cerrada bajo intersecciones finitas. Claramente $\gamma_{n+1}$ es numerable y satisface las condiciones (i)-(vi).

Observemos que la familia $\gamma=\bigcup_{n \in \omega} \gamma_{n}$ es numerable y satisface las condiciones (a) y (b) del lema 2.2.6. Por lo tanto, $N=\bigcap\left\{V \cap V^{-1}: V \in \gamma\right\}$ es un subgrupo invariante de $G$. Consideremos el grupo algebraico $G / N$. Sea 
$p: G \rightarrow G / N$ el homomorfismo canónico. Hagamos $\mathcal{B}=\{p(V): V \in \gamma\}$.

Afirmamos que la familia $\mathcal{B}$ cumple las siguientes propiedades:

(1) para cada $A, B \in \mathcal{B}$, existe $C \in \mathcal{B}$ tal que $C \subseteq A \cap B$;

(2) para cada $A \in \mathcal{B}$, existe $B \in \mathcal{B}$ tal que $B^{2} \subseteq A$;

(3) para cada $A \in \mathcal{B}$ y $a \in A$, existe $B \in \mathcal{B}$ tal que $a B \subseteq A$;

(4) $\mathcal{B}$ está subordinada a cada $A \in \mathcal{B}$.

En efecto, la condición (1) se cumple porque $\gamma$ es cerrada bajo intersecciones finitas. La parte (2) es consecuencia de (iv). La condición (3) se satisface por (vi). Por último, la parte (4) se sigue de (v).

Sea $H=G / N$. De la afirmación podemos concluir que la familia $\mathcal{B}$ es una base local de la identidad para una topología $\tau$ en $H$ tal que $(H, \tau)$ es un grupo paratopológico. Como $\mathcal{B}$ es numerable, $(H, \tau)$ es primero numerable. Veamos que el grupo paratopológico $(H, \tau)$ es $T_{0}$. Sea $y \in H \backslash\left\{e^{\prime}\right\}$ donde $e^{\prime}$ es la identidad de $H$. Tomemos $x \in G$ tal que $p(x)=y$. Como $y \neq e^{\prime}$, tenemos que $x \notin N=\bigcap\left\{V \cap V^{-1}: V \in \gamma\right\}$. Por lo tanto, existe $V \in \gamma$ tal que $x \notin V \cap V^{-1}$. Tomemos $W \in \gamma$ tal que $W^{2} \subseteq V$. Probemos que $y \notin p(W) \cap p(W)^{-1}$, de lo contrario existen $u, v \in W$ tales que $y=p(x)=p(u)=p(v)^{-1}$, es decir, $x u^{-1}, x v \in N$. En consecuencia $x \in N u \cap N v^{-1} \subseteq W^{2} \cap W^{-2} \subseteq V \cap V^{-1}$, lo cual es una contradicción. De lo anterior se sigue que $(H, \tau)$ es $T_{0}$.

Finalmente, tomemos $U \in \gamma$ tal que $U^{2} \subseteq U_{0}^{*}$ y hagamos $V_{0}=p(U)$. Entonces $V_{0}$ es una vecindad abierta de $e^{\prime}$ en $H$ y $p^{-1}\left(V_{0}\right)=U N \subseteq U^{2} \subseteq$ $U_{0}^{*} \subseteq U_{0}$.

En la sección 1.3 se definió el grupo topológico asociado a un grupo paratopológico $G$. De este concepto y el de $\omega$-estrecho (ver definición 2.2.7) surge el siguiente:

Definición 2.2.9. Un grupo paratopológico $G$ es totalmente $\omega$-estrecho si su grupo topológico asociado $G^{*}$ es $\omega$-estrecho.

Teorema 2.2.10. Sea $G$ un grupo paratopológico $T_{0}$. Entonces $G$ es topológicamente isomorfo a un subgrupo de un producto de grupos paratopológicos $T_{0}$ segundo numerables si y sólo si es $G$ totalmente $\omega$-estrecho.

Demostración. Supongamos que $G$ es un subgrupo de $\Pi=\prod_{i \in I} H_{i}$ donde cada $H_{i}$ es un grupo paratopológico $T_{0}$ segundo numerable. Por el lema 1.3.3, el grupo topológico asociado $H_{i}^{*}$ a $H_{i}$ es Hausdorff segundo numerable y, por lo tanto, $\omega$-estrecho. Como la clase de grupos topológicos $\omega$-estrechos es 
cerrada bajo productos y subgrupos, podemos concluir que $G^{*}$ es $\omega$-estrecho, es decir, $G$ es totalmente $\omega$-estrecho.

Ahora supongamos que $G$ es un grupo paratopológico $T_{0}$ totalmente $\omega$ estrecho. Cada grupo paratopológico totalmente $\omega$-estrecho es $\omega$-balanceado (ver [30, Proposition 3.8]). Aplicando el teorema anterior tenemos que $G$ es proyectivamente $T_{0}$ primero numerable. Veamos que $G$ es proyectivamente $T_{0}$ segundo numerable. Sea $U \in \mathcal{N}(e)$. Como $G$ es proyectivamente $T_{0}$ primero numerable, existe un homomorfismo continuo $p: G \rightarrow H$ sobre un grupo paratopológico $T_{0}$ primero numerable $H$ tal que $p^{-1}(V) \subseteq U$ para alguna vecindad abierta $V$ de la identidad en $H$. Sean $G^{*}$ y $H^{*}$ los grupos topológicos asociados a $G$ y $H$, respectivamente. El homomorfismo $p: G^{*} \rightarrow H^{*}$ es continuo. Como $G^{*}$ es $\omega$-estrecho y $H^{*}$ es la imagen de $G^{*}$ bajo $p$, entonces $H^{*}$ es $\omega$-estrecho. Por el lema 1.3.3, $H^{*}$ es un grupo topológico Hausdorff primero numerable. En [31] se prueba que todo grupo topológico $\omega$-estrecho primero numerable es segundo numerable. Como $H$ es imagen continua de $H^{*}$, el espacio $H$ tiene una red numerable. Todo grupo paratopológico primero numerable con red numerable es segundo numerable (ver [5, Proposition 2.13]). Por lo tanto $H$ es $T_{0}$ segundo numerable. Aplicando el corolario 2.2.2, tenemos que $G$ es topológicamente isomorfo a un subgrupo de un producto de grupos paratopológicos $T_{0}$ segundo numerables.

Observemos que para el caso de grupos paratopológicos $T_{0}$ los enunciados de los dos teoremas anteriores son muy similares a los enunciados de los teoremas de Katz y Guran. Para grupos paratopológicos $T_{1}$, utilizamos el concepto de número de simetría.

Teorema 2.2.11. Sea $G$ un grupo paratopológico $T_{1}$. Entonces $G$ es topológicamente isomorfo a un subgrupo de un producto de grupos paratopológicos $T_{1}$ primero numerables si y sólo si es $\omega$-balanceado y $\operatorname{Sm}(G) \leq \omega$.

Demostración. Supongamos que $G$ es un subgrupo de $\Pi=\prod_{i \in I} H_{i}$ donde $H_{i}$ es un grupo paratopológico $T_{1}$ primero numerable para cada $i \in I$. Como cada $H_{i}$ es primero numerable, $H_{i}$ es $\omega$-balanceado y, por lo tanto, el producto $\Pi$ también es $\omega$-balanceado. Como cada $H_{i}$ es $T_{1}$ primero numerable, $H_{i}$ tiene pseudocarácter numerable. Por la proposición 2.1.3 tenemos que $\operatorname{Sm}\left(H_{i}\right) \leq \omega$ para cada $i \in I$. Aplicando las proposiciones 2.1 .2 y 2.1.4, podemos concluir que $\operatorname{Sm}(G) \leq S m(\Pi) \leq \omega$.

Ahora supongamos que $G$ es un grupo paratopológico $\omega$-balanceado $T_{1}$ con $\operatorname{Sm}(G) \leq \omega$. Sea $U_{0} \in \mathcal{N}(e)$. Tenemos que definir un homomorfismo con- 
tinuo sobreyectivo $p: G \rightarrow H$, donde $H$ es un grupo paratopológico primero numerable $T_{1}$ y encontrar una vecindad abierta $V_{0}$ de la identidad en $H$ tal que $p^{-1}\left(V_{0}\right) \subseteq U_{0}$.

Denotemos por $\mathcal{N}^{*}(e)$ la subfamilia de $\mathcal{N}(e)$ la cual consiste de conjuntos $\omega$-buenos. Por el lema 2.2.3, el conjunto $\mathcal{N}^{*}(e)$ es una base local de $e$ en $G$. Definamos inductivamente una sucesión $\left\{\gamma_{n}: n \in \omega\right\}$ de la siguiente forma.

Sea $U_{0}^{*} \in \mathcal{N}^{*}(e)$ tal que $U_{0}^{*} \subseteq U_{0}$. Hagamos $\gamma_{0}=\left\{U_{0}^{*}\right\}$. Supongamos que para algún $n \in \omega$ hemos definido $\gamma_{0}, \ldots, \gamma_{n}$ de tal forma que para cada $k \leq n$ se satisface:

(i) $\gamma_{k} \subseteq \mathcal{N}^{*}(e)$ y $\left|\gamma_{k}\right| \leq \omega$;

(ii) $\gamma_{k-1} \subseteq \gamma_{k}$;

(iii) $\gamma_{k}$ es cerrada bajo intersecciones finitas;

(iv) para cada $U \in \gamma_{k-1}$, existe $V \in \gamma_{k}$ tal que $V^{2} \subseteq U$;

(v) la familia $\gamma_{k}$ está subordinada a $U$, para cada $U \in \gamma_{k-1}$;

(vi) para cada $U \in \gamma_{k-1}$ y $x \in U$, existe $V \in \gamma_{k}$ tal que $x V \subseteq U$;

(vii) $\bigcap_{V \in \gamma_{k}} V^{-1} \subseteq U$, para cada $U \in \gamma_{k-1}$.

Como $\gamma_{n}$ es numerable, existe una familia numerable $\lambda_{n, 1} \subseteq \mathcal{N}^{*}(e)$ tal que cada $U \in \gamma_{n}$ contiene el cuadrado de algún elemento $V \in \lambda_{n, 1}$. Por ser $G$ w-balanceado, podemos encontrar una familia numerable $\lambda_{n, 2} \subseteq \mathcal{N}^{*}(e)$ subordinada a cada $U \in \gamma_{n}$. Como $\gamma_{n} \subseteq \mathcal{N}^{*}(e)$, existe una familia numerable $\lambda_{n, 3} \subseteq \mathcal{N}^{*}(e)$ tal que para cada $U \in \gamma_{n}$ y $x \in U$, existe $V \in \lambda_{n, 3}$ la cual satisface $x V \subseteq U$. Por hipótesis $\operatorname{Sm}(G) \leq \omega$ y como la familia $\gamma_{n}$ es numerable, podemos elegir $\lambda_{n, 4} \subseteq \mathcal{N}^{*}(e)$ tal que $\bigcap_{V \in \lambda_{n, 4}} V^{-1} \subseteq U$, para cada $U \in \gamma_{n}$. Sea $\gamma_{n+1}$ la mínima familia que contiene a $\gamma_{n} \cup \bigcup_{i=1}^{4} \lambda_{n, i}$ y es cerrada bajo intersecciones finitas. Claramente $\gamma_{n+1}$ es numerable y satisface las condiciones (i)-(vii).

Observemos que la familia $\gamma=\bigcup_{n \in \omega} \gamma_{n}$ es numerable y satisface las condiciones (a)-(c) del lema 2.2.6. Por lo tanto, $N=\bigcap_{V \in \gamma} V$ es un subgrupo cerrado invariante de $G$. Sea $p: G \rightarrow G / N$ el homomorismo canónico. Haga$\operatorname{mos} \mathcal{B}=\{p(V): V \in \gamma\}$.

Afirmamos que la familia $\mathcal{B}$ cumple las siguientes propiedades:

(1) para cada $A, B \in \mathcal{B}$, existe $C \in \mathcal{B}$ tal que $C \subseteq A \cap B$;

(2) para cada $A \in \mathcal{B}$, existe $B \in \mathcal{B}$ tal que $B^{2} \subseteq A$;

(3) para cada $A \in \mathcal{B}$ y $a \in A$, existe $B \in \mathcal{B}$ tal que $a B \subseteq A$;

(4) $\mathcal{B}$ está subordinada a cada $A \in \mathcal{B}$.

En efecto, la condición (1) Se cumple porque $\gamma$ es cerrada bajo inter- 
secciones finitas. La parte (2) es consecuencia de (iv). La condición (3) se satisface por (vi). Por último, la parte (4) se sigue de (v).

Sea $H=G / N$. De la afirmación podemos concluir que la familia $\mathcal{B}$ es una base local de la identidad para una topología $\tau$ en $H$ tal que $(H, \tau)$ es un grupo paratopológico. Como $\mathcal{B}$ es numerable, $(H, \tau)$ es primero numerable. Veamos que el grupo paratopológico $(H, \tau)$ es $T_{1}$. Sea $y \in H \backslash\left\{e^{\prime}\right\}$. Tomemos $x \in G$ tal que $p(x)=y$. Como $y \neq e^{\prime}$, tenemos que $x \notin N=\bigcap_{V \in \gamma} V$. Por lo tanto, existe $V \in \gamma$ tal que $x \notin V$. Tomemos $W \in \gamma$ tal que $W^{2} \subseteq V$. Probemos que $y \notin p(W)$. De lo contrario existe $u \in W$ tal que $y=p(x)=p(u)$, es decir, $x u^{-1} \in N$. En consecuencia $x \in N u \subseteq W W \subseteq V$, lo cual es una contradicción. De lo anterior se sigue que $(H, \tau)$ es $T_{1}$.

Finalmente, tomemos $U \in \gamma$ tal que $U^{2} \subseteq U_{0}^{*}$ y hagamos $V_{0}=p(U)$. Entonces $V_{0}$ es una vecindad abierta de $e^{\prime}$ en $H$ y $p^{-1}\left(V_{0}\right)=U N \subseteq U^{2} \subseteq$ $U_{0}^{*} \subseteq U_{0}$.

Corolario 2.2.12. Todo grupo paratopológico $T_{1} \omega$-balanceado con pseudocarácter numerable admite un isomorfismo continuo sobre un grupo paratopológico $T_{1}$ primero numerable.

Demostración. Como $G$ tiene pseudocarácter numerable, $\operatorname{Sm}(G) \leq \omega$. Por el teorema 2.2.11, $G$ puede identificarse como un subgrupo de un producto $H=\prod_{i \in I} H_{i}$ de grupos paratopológicos $T_{1}$ primero numerables. Debido a que $G$ tiene pseudocarácter numerable, podemos encontrar un subconjunto numerable $J \subseteq I$ tal que la restricción a $G$ de la proyección $P_{J}: H \rightarrow$ $\prod_{i \in J} H_{i}$ es inyectiva. Por lo tanto $G$ se condensa en el grupo paratopológico $P_{J}(G)$.

Por $[32$, Theorems 2.7, 3.6] y nuestros teoremas 2.2.8 y 2.2.11, los grupos paratopológicos proyectivamente $T_{i}$ primero numerable están caracterizados internamente, para $i=0,1,2,3$.

Problema 2.2.13. Dar una caracterización interna de los grupos paratopológicos proyectivamente $T_{3 \frac{1}{2}}$ primero numerables.

Teorema 2.2.14. Sea $G$ un grupo paratopológico $T_{1}$. Entonces $G$ es topológicamente isomorfo a un subgrupo de un producto de grupos paratopológicos $T_{1}$ segundo numerables si y sólo si es $G$ totalmente $\omega$-estrecho y $\operatorname{Sm}(G) \leq \omega$.

Demostración. Supongamos que $G$ es un subgrupo de $\Pi=\prod_{i \in I} H_{i}$ donde cada $H_{i}$ es un grupo paratopológico $T_{1}$ segundo numerable. Por el lema 1.3.3, 
el grupo topológico asociado $H_{i}^{*}$ a $H_{i}$ es segundo numerable y, por lo tanto, $\omega$ estrecho. Como $\Pi^{*}=\prod_{i \in I} H_{i}^{*}$, el grupo $\Pi^{*}$ es $\omega$-estrecho. El grupo topológico $G^{*}$ asociado a $G$ es $\omega$-estrecho por ser subgrupo del grupo topológico $\Pi^{*}$. La condición $\operatorname{Sm}(G) \leq \omega$ se verifica como en el teorema 2.2.11.

Ahora supongamos que $G$ es totalmente $\omega$-estrecho y $\operatorname{Sm}(G) \leq \omega$. Cada grupo paratopológico totalmente $\omega$-estrecho es $\omega$-balanceado (ver [30, Proposition 3.8]). Aplicando el teorema 2.2.11, tenemos que $G$ es proyectivamente $T_{1}$ primero numerable. Mediante un razonamiento similar al utilizado en el teorema 2.2.10, se prueba que $G$ es proyectivamente $T_{1}$ segundo numerable. Aplicando el corolario 2.2.2, tenemos que $G$ es topológicamente isomorfo a un subgrupo de un producto de grupos paratopológicos $T_{1}$ segundo numerables.

Corolario 2.2.15. Todo grupo paratopológico $T_{1}$ totalmente $\omega$-estrecho con pseudocarácter numerable admite un isomorfismo continuo sobre un grupo paratopológico $T_{1}$ segundo numerable.

Demostración. Se prueba de forma análoga al corolario 2.2.12.

El siguiente ejemplo y el siguiente lema nos servirán para construir un grupo paratopológico con propiedades interesantes.

Ejemplo 2.2.16. Consideremos el grupo aditivo $(\mathbb{R},+)$. Sea $U_{n}=\left(-\frac{1}{n}, \frac{1}{n}\right) \cup$ $(n, \infty)$, para cada $n \in \mathbb{N}$.

Veamos que la familia $\left\{U_{n}: n \in \mathbb{N}\right\}$ satisface lo siguiente:

i) $U_{n+1} \subseteq U_{n}$, para cada $n \in \mathbb{N}$;

ii) $U_{2 n}+U_{2 n} \subseteq U_{n}$, para cada $n \in \mathbb{N}$;

iii) Para cada $n \in \mathbb{N}$ y cada $u \in U_{n}$, existe $m \in \mathbb{N}$ tal que $u+U_{m} \subseteq U_{n}$;

iv) Para cada $n \in \mathbb{N}$ y cada $x \in \mathbb{R}$ se tiene $x+U_{n}-x=U_{n}$.

v) $\bigcap\left\{U_{n}: n \in \mathbb{N}\right\}=\{0\}$.

En efecto:

i) Evidente. 
ii) Tomemos $x, y \in U_{2 n}$. Si $x, y \in\left(-\frac{1}{2 n}, \frac{1}{2 n}\right)$, entonces $x+y \in\left(-\frac{1}{n}, \frac{1}{n}\right) \subseteq$ $U_{n}$. Si $x \in\left(-\frac{1}{2 n}, \frac{1}{2 n}\right)$ y $y \in(2 n, \infty)$, entonces $x+y \in\left(2 n-\frac{1}{2 n}, \infty\right) \subseteq U_{n}$. Por último, si $x, y \in(2 n, \infty)$ se tiene que $x+y \in(4 n, \infty) \subseteq U_{n}$. Por lo tanto $U_{2 n}+U_{2 n} \subseteq U_{n}$.

iii) Sean $n \in \mathbb{N}$ y $u \in U_{n}$. Si $u \in\left(-\frac{1}{n}, \frac{1}{n}\right)$, existe $m>n$ tal que $u+$ $\left(-\frac{1}{m}, \frac{1}{m}\right) \subseteq\left(-\frac{1}{n}, \frac{1}{n}\right)$. Entonces $u+(m, \infty) \subseteq(n, \infty)$ y, por lo tanto, $u+U_{m} \subseteq U_{n}$. Por otro lado, si $u \in(n, \infty)$, podemos encontrar $m \in \mathbb{N}$ tal que $u-\frac{1}{m}>n$. En este caso, $u+U_{m} \subseteq\left(u-\frac{1}{m}, \infty\right) \subseteq(n, \infty) \subseteq U_{n}$.

iv) Se sigue de la conmutatividad de $\mathbb{R}$.

v) Evidente.

De las condiciones i)-iv) podemos concluir que existe una topología $\mu$ en $(\mathbb{R},+)$ tal que $F=(\mathbb{R}, \mu)$ es un grupo paratopológico y la familia $\left\{U_{n}: n \in\right.$ $\mathbb{N}$ \} es una base local de 0 en $F$. La condición v) implica que el espacio $F$ es $T_{1}$. Como $F$ es totalmente $\omega$-estrecho primero numerable, $F$ es un grupo paratopológico segundo numerable ([2, Proposition 3.5]).

Definición 2.2.17. Sean $X$ y $Y$ dos espacios. Una función $f: X \rightarrow Y$ se llama condensación si $f$ es continua y biyectiva.

Lema 2.2.18. Si existe una condensación de un espacio Hausdorff $X$ sobre un espacio Hausdorff primero numerable, entonces para cada $x \in X$, existe una familia $\left\{V_{n}: n \in \omega\right\}$ de vecindades abiertas de $x$ en $X$ tal que $\bigcap_{n \in \omega} \overline{V_{n}}=$ $\{x\}$.

Definición 2.2.19. ([32]) Dado un grupo semitopológico Hausdorff G se define el número de Hausdorff, denotado por $H s(G)$, como el minimo cardinal $\kappa$ tal que para cada vecindad $U$ de e en $G$, existe una familia $\gamma$ de vecindades de e tal que $\bigcap_{V \in \gamma} V V^{-1} \subseteq U y|\gamma| \leq \kappa$.

Utilizando el número de Hausdorff, M. Tkachenko demuestra el siguiente resultado:

Teorema 2.2.20. ([32, Theorem 2.8]) Un grupo paratopológico Hausdorff $G$ es topológicamente isomorfo a un subgrupo de un producto de grupos paratopológicos Hausdorff segundo numerables si y sólo si es $G$ totalmente $\omega$ estrecho y $H s(G) \leq \omega$. 
En [30, Problem 6.2] M. Sanchis y M. Tkachenko plantean la siguiente pregunta:

a) ¿Es cada grupo paratopológico abeliano Hausdorff totalmente $\omega$-estrecho topologicamente isomorfo a un subgrupo de un producto de grupos paratopológicos Hausdorff segundo numerables?

Aplicando el teorema 2.2.20, podemos escribir la pregunta a) de forma equivalente:

¿Cada grupo paratopológico abeliano Hausdorff totalmente $\omega$-estrecho tiene número de Hausdorff numerable?

Todos los grupos paratopológicos Hausdorff totalmente $\omega$-estrechos conocidos hasta ese momento tenían número de Hausdorff numerable. Con base en lo anterior M. Tkachenko plantea la siguiente cuestión (ver [32, Problem 4.1]):

b) ¿Cada grupo paratopológico Hausdorff totalmente $\omega$-estrecho $G$ satisface la desigualdad $H s(G) \leq \omega$ ?

Un grupo paratopológico $G$ es totalmente Lindelöf si su grupo topológico asociado $G^{*}$ es Lindelöf. En [30] M. Sanchis y M. Tkachenko demuestran que un grupo paratopológico Hausdorff totalmente Lindelöf con pseudocarácter numerable se puede condensar en un grupo paratopológico Hausdorff segundo numerable. En un intento de extender este resultado a la clase de grupos paratopológicos totalmente $\omega$-estrechos, los autores redactan la siguiente pregunta (ver [30, Problem 6.3]):

c) ¿Todo grupo paratopológico Hausdorff totalmente $\omega$-estrecho con pseudocarácter numerable se puede condensar en un grupo paratopológico Hausdorff segundo numerable?

Un corolario del teorema 2.2.20 es el siguiente: un grupo paratopológico Hausdorff totalmente w-estrecho con numero de Hausdorff numerable y pseudocarácter numerable se puede condensar en un grupo paratopológico Hausdorff segundo numerable. Por lo tanto, si logramos construir un grupo paratopológico abeliano Hausdorff $H$, totalmente $\omega$-estrecho con pseudocarácter numerable que no se puede condensar en un espacio Hausdorff primero numerable, entonces $H$ tendrá número de Hausdorff no numerable. Esto respondería las preguntas a)-c).

Definición 2.2.21. Un espacio $X$ es funcionalmente Hausdorff si para cada par de puntos distintos $x, y \in X$, existe una función continua $f: X \rightarrow[0,1]$ tal que $f(x)=0$ y $f(y)=1$. 
El siguiente ejemplo responde en forma negativa a las preguntas a)-c).

Ejemplo 2.2.22. Existe un grupo paratopológico abeliano $H$, funcionalmente Hausdorff, totalmente $\omega$-estrecho con pseudocarácter numerable que no se puede condensar en un espacio Hausdorff primero numerable. En consecuencia, $H s(H)>\omega$.

Consideremos el grupo del círculo $\mathbb{T}$ con su topología usual y notación aditiva. Sea $F$ el grupo paratopológico del ejemplo 2.2.16. Sea $K=F \times \mathbb{T}^{\omega_{1} \backslash\{0\}}$. Tomemos un conjunto $\left\{a_{\alpha}: \alpha \in \omega_{1} \backslash\{0\}\right\} \subseteq \mathbb{R}^{+}$linealmente independiente en $(\mathbb{R},+)$. Sea $t \in \mathbb{T}$ un elemento de orden infinito. Para cada $\alpha \in \omega_{1} \backslash\{0\}$ definamos el elemento $x_{\alpha} \in K$ de la siguiente manera:

$$
x_{\alpha}(\beta)= \begin{cases}a_{\alpha}, & \beta=0 \\ t, & \beta=\alpha ; \\ 0, & \text { en otro caso. }\end{cases}
$$

Sea $H$ el subgrupo de $K$ generado por el conjunto $\left\{x_{\alpha}: \alpha \in \omega_{1} \backslash\{0\}\right\}$. Veamos que el grupo paratopológico $H$ es funcionalmente Hausdorff. Tomemos $x \in$ $H \backslash\{0\}$; entonces $x=n_{1} x_{\alpha_{1}}+n_{2} x_{\alpha_{2}}+\cdots+n_{k} x_{\alpha_{k}}$ con $k \in \mathbb{N}, \alpha_{1}, \alpha_{2}, \ldots, \alpha_{k} \in$ $\omega_{1} \backslash\{0\}$ distintos y $n_{i} \in \mathbb{Z} \backslash\{0\}$ para cada $i \leq k$. Observemos que $x\left(\alpha_{1}\right)=n_{1} t$. Como $n_{1} \in \mathbb{Z} \backslash\{0\}$ y $t$ es de orden infinito, $n_{1} t \neq 0_{\mathbb{T}}$. Sea $p_{\alpha_{1}}: K \rightarrow \mathbb{T}_{\alpha_{1}}$ la proyección de $K$ en el factor $\alpha_{1}$. Denotemos por $g$ a la restricción de $p_{\alpha_{1}}$ a $H$. Como $\mathbb{T}$ es Tychonoff, existe una función continua $h: \mathbb{T} \rightarrow[0,1]$ tal que $h\left(0_{\mathbb{T}}\right)=0$ y $h\left(n_{1} t\right)=1$. Si $f=h \circ g$, entonces la función $f$ es continua $\mathrm{y}$, además, $f\left(0_{H}\right)=0$ y $f(x)=h(g(x))=h\left(n_{1} t\right)=1$.

Sabemos que los espacios $F$ y $\mathbb{T}$ son $T_{1}$ segundo numerables. Aplicando el teorema 2.2.14, tenemos que $H$ es totalmente $\omega$-estrecho y $\operatorname{Sm}(H) \leq \omega$.

El conjunto $Z=\left(\left\{0_{F}\right\} \times \mathbb{T}^{\omega_{1} \backslash\{0\}}\right) \cap H$ es un conjunto $G_{\delta}$ en $H$. Si $n_{1} x_{\alpha_{1}}+$ $n_{2} x_{\alpha_{2}}+\cdots+n_{k} x_{\alpha_{k}}=x \in Z$, entonces $0_{F}=x(0)=n_{1} a_{\alpha_{1}}+n_{2} a_{\alpha_{2}}+\cdots+n_{k} a_{\alpha_{k}}$ y como el conjunto $\left\{a_{\alpha}: \alpha \in \omega_{1} \backslash\{0\}\right\}$ es linealmente independiente, tenemos que $n_{i}=0$ para cada $i \leq k \mathrm{y}$, por lo tanto $x=0_{H}$. De lo anterior, se sigue que $H$ tiene pseudocarácter numerable.

Para probar que $H$ no se puede condensar en un espacio Hausdorff primero numerable, necesitamos demostrar la siguiente afirmación.

Afirmación. Si $U \in \mathcal{N}_{K}\left(0_{K}\right)$ es un abierto canónico en $K$ y $V=U \cap H$, entonces $x_{\beta} \in \bar{V}^{H}$ para cada $\beta \in \omega_{1} \backslash\{0\}$ tal que $\beta \notin \operatorname{supp}(U)$. 
Demostración de la afirmación. Sean $U, V$ y $x_{\beta}$ como en el enunciado de la afirmación. Por hipótesis, $U=\prod\left\{P_{\alpha}: \alpha \in \omega_{1}\right\}$. Sea $O \in \mathcal{N}_{K}\left(0_{K}\right)$ un abierto canónico en $K$ tal que $O \subseteq U$ y $0 \in \operatorname{supp}(O)$. Hagamos $O=\prod\left\{O_{\alpha}: \alpha \in \omega_{1}\right\}$ y $\operatorname{supp}(O)=\left\{0, \alpha_{1}, \alpha_{2}, \ldots, \alpha_{k}\right\}$. Tenemos que $O_{\alpha_{i}} \subseteq P_{\alpha_{i}}$ para cada $\alpha \in \omega_{1}$ y $\operatorname{supp}(U) \subseteq \operatorname{supp}(O)$. Existe $n_{0} \in \mathbb{N}$ tal que $U_{n_{0}} \subseteq O_{0}$. Sea $r$ un entero positivo tal que $r a_{\alpha_{1}}>n_{0}$. Si $z \in \mathbb{T}$ es de orden infinito, entonces el conjunto $\{n z: n \in \mathbb{N}\}$ es denso en $\mathbb{T}$. Para cada $i \leq k$ existe un entero positivo $r_{i}$ tal que $r_{i} t \in O_{\alpha_{i}}$, podemos tomar $r_{1} \geq r$. Sea $y=r_{1} x_{\alpha_{1}}+r_{2} x_{\alpha_{2}}+\cdots+r_{k} x_{\alpha_{k}}$. Recordemos que $a_{\alpha_{i}}>0$ para cada $i \leq k$, por lo tanto

$y(0)=r_{1} a_{\alpha_{1}}+\cdots+r_{k} a_{\alpha_{k}}>r_{1} a_{\alpha_{1}} \geq r a_{\alpha_{1}}>n_{0}$, es decir, $y(0) \in U_{n_{0}} \subseteq O_{0}$.

Además, $y\left(\alpha_{i}\right)=r_{i} t \in O_{\alpha_{i}}$, para cada $i \leq k$.

De lo anterior podemos concluir que $y \in O \cap H$.

Tomemos $\beta<\omega_{1} \backslash\{0\}$ tal que $\beta \notin \operatorname{supp}(U)$. Sea $z=x_{\beta}+y$. Entonces para cada $i \leq k$ tal que $\alpha_{i} \in \operatorname{supp}(U) \backslash\{0\}$ se tiene

$$
z\left(\alpha_{i}\right)=y\left(\alpha_{i}\right)=r_{i} t \in O_{\alpha_{i}} \subseteq P_{\alpha_{i}} .
$$

Además, $z(0)=r_{1} a_{\alpha_{1}}+\cdots+r_{k} a_{\alpha_{k}}+a_{\beta}>r_{1} a_{\alpha_{1}} \geq r a_{\alpha_{1}}>n_{0}$. Por lo tanto, $z(0) \in U_{n_{0}} \subseteq O_{0} \subseteq P_{0}$.

De modo que, $z \in U \cap H=V$ y $\left(x_{\beta}+W\right) \cap V \neq \emptyset$, donde $W=O \cap H \ni y$. Se sigue que $x_{\beta} \in \bar{V}^{H}$ y la afirmación queda probada.

Supongamos que existe una condensación de $H$ sobre un espacio Hausdorff primero numerable. Por el lema 2.2.18, existe $\left\{V_{n}: n \in \omega\right\} \subseteq \mathcal{N}_{H}\left(e_{H}\right)$ tal que $\bigcap_{n \in \omega}{\overline{V_{n}}}^{H}=\left\{e_{H}\right\}$. Para cada $n \in \omega$, existe $U_{n} \in \mathcal{N}_{K}(0)$ abierto canónico en $K$ tal que $U_{n} \cap H \subseteq V_{n}$. El conjunto $S=\bigcup\left\{\operatorname{supp}\left(U_{n}\right): n \in \omega\right\} \subseteq \omega_{1}$ es numerable. Tomemos $\alpha \in \omega_{1} \backslash\{0\}$ tal que $\alpha \notin S$. Aplicando la afirmación, tenemos que $x_{\alpha} \in{\overline{V_{n}}}^{H}$, para cada $n \in \omega$, es decir, $x_{\alpha} \in \bigcap_{n \in \omega}{\overline{V_{n}}}^{H}=\left\{0_{H}\right\}$, lo cual es una contradicción. Podemos concluir que $H$ no se puede condensar en un espacio Hausdorff primero numerable.

El ejemplo anterior tiene número de simetría númerable. A continuación mostramos que la condición "número de simetría numerable" no puede ser omitida en el teorema 2.2.14.

Ejemplo 2.2.23. Existe un grupo paratopológico abeliano funcionalmente Hausdorff totalmente w-estrecho con número de simetría no numerable.

Dado $x \in \mathbb{Z}$, hagamos $R_{x}=\{y \in \mathbb{Z}: y \geq x\}$. La familia $\left\{R_{x}: x \in \mathbb{Z}\right\}$ 
es una base para una topología $\sigma$ en $\mathbb{Z}$ tal que $G=(\mathbb{Z}, \sigma)$ es un grupo paratopológico $T_{0}$ segundo numerable. Una base local para el cero en $G$ es la familia $\left\{R_{0}\right\}$.

Consideremos al grupo del círculo $\mathbb{T}$ con notación aditiva y su topología usual. Sea $t \in \mathbb{T}$ un elemento de orden infinito. Hagamos $K=G \times \mathbb{T}^{\omega_{1} \backslash\{0\}}$. Para cada $\alpha \in \omega_{1} \backslash\{0\}$, definamos $x_{\alpha} \in K$ de la siguiente manera:

$$
x_{\alpha}(\beta)= \begin{cases}-1, & \beta=0 \\ t, & \beta=\alpha \\ 0_{\mathbb{T}}, & \text { en otro caso }\end{cases}
$$

Sea $H$ el subgrupo de $K$ generado por el conjunto $\left\{x_{\alpha}: \alpha \in \omega_{1} \backslash\{0\}\right\}$. Con un argumento similar al utilizado en el ejemplo 2.2.22, podemos probar que $H$ es funcionalmente Hausdorff.

Aplicando el teorema 2.2.10, tenemos que $H$ es totalmente $\omega$-estrecho. Ahora, veamos que $H$ tiene número de simetría no numerable. Sean $U=H \cap$ $\left(R_{0} \times \mathbb{T}^{\omega_{1} \backslash\{0\}}\right)$ y $\left\{V_{n}: n \in \omega\right\} \subseteq \mathcal{N}_{H}\left(0_{H}\right)$. Para cada $n \in \omega$, podemos encontrar un abierto canónico $U_{n}$ en $K$ tal que $U_{n} \cap H \subseteq V_{n}$. Sea $P=\bigcup_{n \in \omega} \operatorname{supp}\left(U_{n}\right)$. El conjunto $P$ es numerable y está contenido en $\omega_{1}$, así que podemos tomar $\alpha \in \omega_{1} \backslash\{0\}$ tal que $\alpha \notin P$. Por lo tanto, $x_{\alpha} \in \bigcap_{n \in \omega}\left(U_{n}^{-1} \cap H\right) \subseteq \bigcap_{n \in \omega} V_{n}^{-1}$. Sin embargo, $x_{\alpha} \notin U$. De lo anterior, podemos concluir que $H$ tiene número de simetría no numerable.

A partir de las definiciones de número de Hausdorff y número de simetría se puede concluir que para un grupo paratopológico Hausdorff $G$ se tiene $\operatorname{Sm}(G) \leq H s(G)$. Si se satisface la desigualdad $H s(G) \leq \omega$, entonces $\operatorname{Sm}(G)=H s(G)$. Denotemos por $\mathcal{C}_{\omega}$ a la clase de grupos paratopológicos Hausdorff que cumplen $H s(G) \leq \omega$. Por las proposiciones 2.1-2.4 en [32], podemos concluir que $\mathcal{C}_{\omega}$ contiene a los grupos paratopológicos Hausdorff primero numerables, Lindelöf, grupos topológicos, la clase $\mathcal{C}_{\omega}$ es cerrada bajo subgrupos y productos arbitrarios. En general, la igualdad $S m(H)=H s(H)$ no se cumple para un grupo paratopológico $H$. Basta tomar el grupo $H$ del ejemplo 2.2.23. Con base en las observaciones anteriores surge la siguiente pregunta.

Problema 2.2.24. ¿Qué propiedades debe tener un grupo paratopológico $G$ para que se satisfaga la igualdad $\operatorname{Sm}(G)=H s(G)$ ? 


\section{Capítulo 3}

\section{Funciones cardinales}

En este capítulo probamos que todo grupo paratopológico regular totalmente $\omega$-estrecho tiene índice de regularidad numerable (ver teorema 3.1.3). Este resultado nos ayuda a responder una pregunta planteada por M. Tkachenko en [34].

En el corolario 3.2.10 demostramos que cada grupo paratopológico Hausdorff con $\pi$-carácter numerable tiene diagonal $G_{\delta}$ de rango infinito. Este hecho da una respuesta positiva a una pregunta planteada por A. Arhangel'skii y A. Bella en [2, Problem 3].

En el teorema 3.3.4 probamos que si $G$ es un grupo paratopológico Hausdorff Lindelöf con pseudocarácter numerable, entonces $G$ se puede condensar sobre un espacio métrico segundo numerable. Este teorema resuelve un problema propuesto por M. Tkachenko en [33].

\section{1. Índice de regularidad y de estrechez}

Definición 3.1.1. ([32]) el índice de regularidad de un grupo paratopológico regular $G$, denotado por $\operatorname{Ir}(G)$, es el mínimo cardinal $\kappa$ tal que para cada vecindad $U$ de e en $G$, podemos encontrar una vecindad $V \in \mathcal{N}(e)$ y una familia $\gamma \subseteq \mathcal{N}(e)$ tales que $\bigcap_{W \in \gamma} V W^{-1} \subseteq U y|\gamma| \leq \kappa$.

Utilizando el índice de regularidad M. Tkachenko caracteriza internamente a los subgrupos de productos de grupos paratopológicos regulares segundo numerables: 
Teorema 3.1.2. ([32]) Un grupo paratopológico regular $G$ es topológicamente isomorfo a un subgrupo de un producto de grupos paratopológicos regulares segundo numerables si y sólo si $\operatorname{Ir}(G) \leq \omega$ y $G$ es totalmente $\omega$-estrecho.

En [34, Problem 7.4], Tkachenko plantea la siguiente pregunta:

A) Sea $G$ un grupo paratopológico Tychonoff totalmente $\omega$-estrecho. ¿Es $G$ topológicamente isomorfo a un subgrupo de un producto de grupos paratopológicos regulares segundo numerables?

Por el Teorema 3.1.2, la pregunta anterior se puede reescribir de la siguiente manera:

¿Todo grupo paratopológico Tychonoff totalmente $\omega$-estrecho tiene índice de regularidad numerable?

El siguiente resultado responde en forma positiva la pregunta A).

Teorema 3.1.3. Sea $G$ un grupo paratopológico regular totalmente $\tau$-estrecho. Entonces $\operatorname{Ir}(G) \leq \tau$. En particular, todo grupo paratopológico regular totalmente $\omega$-estrecho tiene indice de regularidad numerable.

Demostración. Tomemos $U \in \mathcal{N}(e)$. Como $G$ es un grupo paratopológico regular, podemos encontrar $V \in \mathcal{N}(e)$ tal que $V \overline{V^{2}} \subseteq U$. Por hipótesis, existe un subconjunto $F \subseteq G^{*}$ que satisface $|F| \leq \tau$ y $\left(V \cap V^{-1}\right) F=G^{*}$. Aquí $G^{*}$ es el grupo topológico asociado a $G$ (ver sección 1.3). Hagamos $A=F \cap \overline{V^{2}}$ y $B=F \backslash A$. Si $a \in A$, entonces $V a \subseteq V \overline{V^{2}} \subseteq U$, por lo tanto, $\left(V \cap V^{-1}\right) A \subseteq U$. Se sigue que $G \backslash U \subseteq\left(V \cap V^{-1}\right) B$. Para cada $b \in B$, existe $W_{b} \in \mathcal{N}(e)$ tal que $b W_{b} \cap V^{2}=\emptyset$, equivalentemente, $V^{-1} b \cap V W_{b}^{-1}=\emptyset$. Afirmamos que $\bigcap_{b \in B} V W_{b}^{-1} \subseteq U$. De lo contrario, podemos encontrar $z \in\left(\bigcap_{b \in B} V W_{b}^{-1}\right) \backslash U$. Como $G \backslash U \subseteq\left(V \cap V^{-1}\right) B$, podemos escoger $b \in B$ tal que $z \in V^{-1} b$. Por lo tanto, $z \in V^{-1} b \cap V W_{b}^{-1}$, lo cual contradice la elección de $W_{b}$. De lo anterior, concluimos que $\bigcap_{b \in B} V W_{b}^{-1} \subseteq U$. Como $|B| \leq \tau$, tenemos que $\operatorname{Ir}(G) \leq \tau$.

No sabemos si el resultado anterior se puede generalizar a grupos paratopológicos regulares $\omega$-estrechos.

Problema 3.1.4. ¿Cada grupo paratopológico regular $\omega$-estrecho tiene índice de regularidad numerable?

El resultado anterior nos permite reformular el teorema 3.1.2 como sigue: 
Corolario 3.1.5. Un grupo paratopológico regular $G$ es topológicamente isomorfo a un subgrupo de un producto de grupos paratopológicos regulares segundo numerables si y sólo si $G$ es totalmente w-estrecho.

La siguiente pregunta planteada por M. Tkachenko, sigue sin responderse.

Problema 3.1.6. ([33]) Sea $G$ un grupo paratopológico regular (primero numerable). ¿Es $G$ completamente regular?, ¿es $G$ funcionalmente Hausdorff?

En los siguientes dos corolarios obtenemos resultados relacionados con el problema anterior.

Corolario 3.1.7. Todo grupo paratopológico regular totalmente w-estrecho es completamente regular.

Demostración. Es consecuencia inmediata del Corolario 3.1.5.

Corolario 3.1.8. Todo grupo paratopológico Hausdorff totalmente w-estrecho es funcionalmente Hausdorff.

Demostración. Sea $G$ un grupo paratopológico Hausdorff totalmente $\omega$-estrecho. Por el teorema 1.4.1, la regularización $r(G)$ de $G$ es un grupo paratopológico regular. Como la topología en $r(G)$ es más débil que la topología en $G$, tenemos que $r(G)$ es totalmente $\omega$-estrecho. Aplicando el Corolario 3.1.7, podemos concluir que $r(G)$ es completamente regular. Se sigue de lo anterior que $G$ es funcionalmente Hausdorff.

Proposición 3.1.9. Si G es grupo paratopológico regular totalmente $\omega$-estrecho con pseudocarácter numerable, entonces $G$ admite un isomorfismo continuo sobre un grupo paratopológico metrizable separable.

Demostración. Por el Teorema 3.1.5, G puede identificarse como un subgrupo de un producto $H=\prod_{i \in I} H_{i}$ de grupos paratopológicos metrizables separables. Debido a que $G$ tiene pseudocarácter numerable, podemos encontrar un subconjunto numerable $J \subseteq I$ tal que la restricción a $G$ de la proyección $p_{J}: H \rightarrow \prod_{i \in J} H_{i}$ es inyectiva. Por lo tanto $G$ admite un isomorfimo continuo sobre el grupo paratopológico metrizable separable $p_{J}(G)$.

En [30] se plantea el problema: ¿cada grupo paratopológico Hausdorff totalmente $\omega$-estrecho con pseudocarácter numerable admite un isomorfismo continuo sobre un grupo paratopológico Hausdorff segundo numerable? El ejemplo 2.2.22, responde en forma negativa a esta pregunta. A continación damos una caracterización de los grupos paratopológicos correspondientes. 
Proposición 3.1.10. Sea $G$ un grupo paratopológico Hausdorff totalmente $\omega$-estrecho con identidad e. Entonces son equivalentes:

a) G admite un isomorfismo continuo sobre un grupo paratopológico metrizable separable;

b) existe una familia numerable $\gamma \subseteq \mathcal{N}(e)$ tal que $\bigcap_{V \in \gamma} \bar{V}=\{e\}$.

Demostración. Claramente a) implica b). Para probar que b) implica a) basta con tomar la regularización $r(G)$ de $G$ y aplicar la proposición anterior.

Definición 3.1.11. Dado un espacio $X$, el número Lindelöf débil de $X$, denotado por $w l(X)$, es el mínimo cardinal $\kappa \geq \omega$ tal que cada cubierta abierta de $X$ contiene una subfamilia $\lambda$ tal que $\bigcup \lambda$ es denso en $X$ y $|\lambda| \leq \kappa$. En particular, $X$ es débilmente Lindelöf si $w l(X) \leq \omega$.

Denotemos por $c(X), d(X)$ y $l(X)$ a la celularidad, densidad y el número de Lindelöf de un espacio $X$. Claramente $w l(X) \leq c(X) \leq d(X)$ y $w l(X) \leq$ $l(X)$. En [32], se demuestra que cada grupo paratopológico regular $G$ satisface $\operatorname{Ir}(G) \leq l(G)$. En el siguiente resultado mejoramos esta cota para el índice de regularidad.

Teorema 3.1.12. Para cada grupo paratopológico regular $G$ se satisface la desigualdad $\operatorname{Ir}(G) \leq w l(G)$.

Demostración. Sea $U \in \mathcal{N}(e)$. Como $G$ es un grupo paratopológico regular, podemos encontrar $W, V \in \mathcal{N}(e)$ tales que $\bar{W} \subseteq U$ y $\overline{V^{2}} \subseteq W$. Para cada $x \in G \backslash \overline{V^{2}}$, existe $W_{x} \in \mathcal{N}(e)$ tal que $x W_{x}^{2} \cap V^{2}=\emptyset$, o equivalentemente, $V^{-1} x W_{x} \cap V W_{x}^{-1}=\emptyset$. Como la familia $\left\{x W_{x}: x \in G \backslash \overline{V^{2}}\right\}$ cubre a $G \backslash W$, la familia $\{W\} \cup\left\{x W_{x}: x \in G \backslash \overline{V^{2}}\right\}$ es una cubierta abierta de $G$, por lo tanto, existe $S \subseteq G \backslash \overline{V^{2}}$ tal que $|S| \leq w l(G)$ y $W \cup \bigcup_{x \in S} x W_{x}$ es denso en $G$. Sea $O=\bigcup_{x \in S} x W_{x}$. Veamos que $G \backslash U \subseteq \bar{O}$. Tomemos $z \in G \backslash U$ y $P$ un abierto que contiene a $z$. Como $\bar{W} \subseteq U$, podemos suponer que $P$ no intersecta a $W$. La densidad de $W \cup O$ en $G$, implica que $P \cap O \neq \emptyset$. De lo anterior se sigue que $z \in \bar{O}$, para cada $z \in G \backslash U$. Por lo tanto, $G \backslash U \subseteq \bar{O} \subseteq V^{-1} O=\bigcup_{x \in S} V^{-1} x W_{x}$. Como $V^{-1} x W_{x} \cap V W_{x}^{-1}=\emptyset$, para cada $x \in S$, tenemos que $\bigcap_{x \in S} V W_{x}^{-1} \subseteq U$, lo cual prueba que $\operatorname{Ir}(G) \leq w l(G)$.

Corolario 3.1.13. Sea $G$ un grupo paratopológico regular. Si $G$ satisface una de las siguientes condiciones, entonces $\operatorname{Ir}(G) \leq \omega$ : 
a) G tiene celularidad numerable;

b) $G$ es separable;

c) G contiene a un subespacio denso Lindelöf.

Corolario 3.1.14. Cada grupo paratopológico regular precompacto tiene índice de regularidad numerable.

Demostración. Sea $G$ un grupo paratopológico regular precompacto. Por [8, Corollary 3], $G$ tiene celularidad numerable. Aplicando el corolario anterior, podemos concluir que $\operatorname{Ir}(G) \leq \omega$.

En lo sucesivo utilizaremos el siguiente resultado:

Teorema 3.1.15. ([32]) Un grupo paratopológico regular $G$ es topológicamente isomorfo a un subgrupo de un producto de grupos paratopológicos regulares primero numerables si y sólo si $\operatorname{Ir}(G) \leq \omega$ y $G$ es $\omega$-balanceado.

Corolario 3.1.16. Sea $G$ un grupo paratopológico regular $\omega$-balanceado. Si $G$ satisface una de las siguientes condiciones, entonces $G$ es topológicamente isomorfo a un subgrupo de un producto de grupos paratopológicos regulares primero numerables.

a) G tiene celularidad numerable;

b) $G$ es separable;

c) G contiene a un subespacio denso Lindelöf;

d) $G$ es precompacto.

Proposición 3.1.17. Sea $G$ un subgrupo regular de un grupo paratopológico $T_{1} \sigma$-compacto $H$. Entonces $G$ es topológicamente isomorfo a un subgrupo de un producto de grupos paratopológicos metrizables. En consecuencia $G$ es completamente regular.

Demostración. Por el lema 1.3.3, el grupo topológico asociado $H^{*}$ es Hausdorff $\sigma$-compacto. Lo anterior implica que $H^{*}$ es $\omega$-estrecho. Como $G^{*}$ es un subgrupo del grupo topológico $H^{*}$, el grupo topológico $G^{*}$ es $\omega$-estrecho (ver [6, Theorem 3.4.4]), es decir, $G$ es totalmente $\omega$-estrecho. La conclusión se sigue de los corolarios 3.1.5 y 3.1.7. 
Lema 3.1.18. Si $\kappa$ es un cardinal tal que $\kappa \leq 2^{\omega}$ y $\mathbb{T}$ es el grupo del círculo con su topología usual, entonces existe $x \in \mathbb{T}^{\kappa}$ tal que el subgrupo generado por $\{x\}$ es denso en $\mathbb{T}^{\kappa}$. Además, $x(\alpha)$ es de orden infinito para cada $\alpha \in \kappa$.

Demostración. Por [16, Corollary 25.15], existe $x \in \mathbb{T}^{\kappa}$ tal que el subgrupo generado por $\{x\}$ es denso en $\mathbb{T}^{\kappa}$. Para cada $\alpha \in \kappa$, sea $p_{\alpha}: \mathbb{T}^{\kappa} \rightarrow \mathbb{T}$ la proyección sobre el factor $\alpha$. Como $p_{\alpha}$ es un homomorfismo continuo, el subgrupo generado por $\{x(\alpha)\}$ es denso en $\mathbb{T}$, lo cual implica que $x(\alpha)$ es de orden infinito.

Por el teorema 3.1.12 podemos concluir que para cada grupo paratopológico regular $G$ se satisface $\operatorname{Sm}(G) \leq H s(G) \leq w l(G)$. El siguiente ejemplo muestra que este resultado no se puede "extender" a grupos paratopológicos Hausdorff, es decir, el número Lindelöf débil de un grupo paratopológico Hausdorff no acota superiormente al número de simetría.

Ejemplo 3.1.19. Existe un grupo paratopológico abeliano funcionalmente Hausdorff totalmente separable (en consecuencia, totalmente débilmente Lindelöf) $H$ con número de simetría no numerable. Además, existe un isomorfismo continuo de $H$ sobre un grupo topológico Hausdorff precompacto.

Demostración. para cada $x \in \mathbb{Z}$, hagamos $R_{x}=\{y \in \mathbb{Z}: y \geq x\}$. La familia $\left\{R_{x}: x \in \mathbb{Z}\right\}$ es base para una topología $\sigma$ en $\mathbb{Z}$ tal que $G=(\mathbb{Z}, \sigma)$ es un grupo paratopológico $T_{0}$ segundo numerable. Una base local para el cero en $G$ es la familia $\left\{R_{0}\right\}$.

Consideremos al grupo del círculo $\mathbb{T}$ con notación aditiva y su topología usual. Por el Lema anterior, existe un elemento $x \in \mathbb{T}^{\omega_{1} \backslash\{0\}}$ tal que el subgrupo generado por $\{x\}$ es denso en $\mathbb{T}^{\omega_{1} \backslash\{0\}}$. Hagamos $K=G \times \mathbb{T}^{\omega_{1} \backslash\{0\}}$. Sea $x_{0} \in K$ tal que $\left.x_{0}\right|_{\mathbb{T}^{\omega_{1} \backslash\{0\}}}=x$ y $x_{0}(0)=0$. Fijemos un elemento $t \in \mathbb{T}$ de orden infinito. Para cada $\alpha \in \omega_{1} \backslash\{0\}$, definamos $x_{\alpha} \in K$ de la siguiente manera:

$$
x_{\alpha}(\beta)= \begin{cases}-1, & \beta=0 \\ t, & \beta=\alpha \\ 0_{\mathbb{T}}, & \text { en otro caso }\end{cases}
$$

Sea $H$ el subgrupo de $K$ generado por el conjunto $X=\left\{x_{\alpha}: \alpha \in \omega_{1}\right\}$. Veamos que $H$ es funcionalmente Hausdorff. Sea $p$ la proyección de $K$ sobre $\mathbb{T}^{\omega_{1} \backslash\{0\}}$ y $\left.p\right|_{H}$ la restricción de $p$ a $H$. Es fácil ver que el conjunto $\left\{\left.x_{\alpha}\right|_{\mathbb{T}^{\omega_{1} \backslash\{0\}}}: \alpha \in \omega\right\}$ 
es independiente en $\mathbb{T}^{\omega_{1} \backslash\{0\}}$, por lo tanto $\left.p\right|_{H}:\left.H \rightarrow p\right|_{H}(H)$ es un isomorfismo continuo. Como $\mathbb{T}^{\omega_{1} \backslash\{0\}}$ es completamente regular, $H$ es funcionalmente Hausdorff. Como $\mathbb{T}^{\omega_{1} \backslash\{0\}}$ es precompacto, el subgrupo $\left.p\right|_{H}(H)$ también es precompacto (ver [6, Proposition 3.7.4]).

El grupo topológico asociado a $K$ es $K^{*}=\mathbb{Z} \times \mathbb{T}^{\omega_{1} \backslash\{0\}}$, donde $\mathbb{Z}$ tiene la topología discreta y $\mathbb{T}$ la topología usual. Sea $F$ el subgrupo de $K$ generado por el conjunto $\left\{x_{\alpha}: \alpha \in \omega\right\}$. Veamos que $F^{*}$ es denso en $K^{*}$. Sea $U=\prod_{\alpha \in \omega_{1}} U_{\alpha}$ un abierto canónico no vacío en $K^{*}$. Podemos suponer que $\operatorname{supp}(U)=\left\{0, \alpha_{1}, \ldots, \alpha_{k}\right\}$ y $U_{0}=\{m\}$, donde $m \in \mathbb{Z}$. Como el grupo generado por $\{x\}$ es denso en $\mathbb{T}^{\omega_{1} \backslash\{0\}}$, existe un entero $r$ tal que $r x\left(\alpha_{i}\right)=r x_{0}\left(\alpha_{i}\right) \in U_{\alpha_{i}}$, para cada $i \leq k$. Tomemos $\beta \in \omega_{1} \backslash \operatorname{supp}(U)$. Consideremos el elemento $z=r x_{0}-m x_{\beta}$. Tenemos que $z(0)=m \in U_{0} \mathrm{y}$ $z\left(\alpha_{i}\right)=r x_{0}\left(\alpha_{i}\right) \in U_{\alpha_{i}}$, para cada $i \leq k$, lo cual implica que $z \in U \cap F^{*}$. Se sigue de lo anterior que $F^{*}$ es denso en $K^{*}$ y por lo tanto, $F^{*}$ es denso en $H^{*}$. Como $F^{*}$ es numerable, podemos concluir que $H^{*}$ es separable.

Sea $M$ el subgrupo de $K$ generado por el conjunto $\left\{x_{\alpha}: \alpha \in \omega_{1} \backslash\{0\}\right\}$. En el ejemplo 2.2.23 se prueba que $\operatorname{Sm}(M)>\omega$. Como $M$ es subgrupo de $H$, entonces $\omega<\operatorname{Sm}(M) \leq S m(H)$.

Por el corolario 3.1.14, todo grupo paratopológico precompacto regular tiene número de simetría numerable. Veamos que la condición "regular" no se puede sustituir por "Hausdorff".

Ejemplo 3.1.20. Existe un grupo paratopológico Hausdorff precompacto con número de simetría no numerable.

Demostración. En [7] se prueba que un grupo paratopológico Hausdorff es un subgrupo de un grupo paratopológico precompacto Hausdorff si y sólo si admite un isomorfismo continuo sobre un grupo topológico precompacto Hausdorff. El grupo $H$ del ejemplo anterior admite un isomorfismo continuo sobre un grupo topológico precompacto Hausdorff. Por lo tanto, $H$ es un subgrupo de un grupo paratopológico precompacto Hausdorff $H_{1}$. Por último, $\omega<\operatorname{Sm}(H) \leq \operatorname{Sm}\left(H_{1}\right)$.

Por la proposición 3.1.9, cada grupo paratopológico regular totalmente débilmente Lindelöf con pseudocarácter numerable admite un isomorfismo continuo sobre un grupo paratopológico Hausdorff segundo numerable. A continuación probamos que este resultado, en general, no es válido en el caso de grupos paratopológicos Hausdorff. 
Ejemplo 3.1.21. Existe un grupo paratopológico abeliano funcionalmente Hausdorff totalmente separable con pseudocarácter numerable que no admite una condensación sobre un espacio primero numerable.

Demostración. Sea $F=(\mathbb{R}, \mu)$ el grupo paratopológico que tiene como base local del cero a la familia $\left\{U_{n}=\left(-\frac{1}{n}, \frac{1}{n}\right) \cup(n, \infty): n \in \mathbb{N}\right\}$; entonces $F$ es $T_{1}$ segundo numerable (ver ejemplo 2.2.16). Consideremos el grupo del círculo $\mathbb{T}$ con notación aditiva y su topología usual. Tomemos un conjunto $A=\left\{a_{\alpha}: \alpha \in \omega_{1}\right\} \subseteq \mathbb{R}^{+}$independiente en $(\mathbb{R},+)$ tal que $0_{\mathbb{R}}$ es un punto de acumulación de $A$ en $\mathbb{R}$ con la topología usual. Tomemos un elemento $x \in$ $\mathbb{T}^{\omega_{1} \backslash\{0\}}$ tal que el subgrupo generado por $\{x\}$ es denso en $\mathbb{T}^{\omega_{1} \backslash\{0\}}$. Hagamos $K=F \times \mathbb{T}^{\omega_{1} \backslash\{0\}}$. Sea $x_{0} \in K$ tal que $x_{0}\left\lceil_{\mathbb{T}^{\omega_{1} \backslash\{0\}}}=x\right.$ y $x_{0}(0)=a_{0}$. Fijemos un elemento $t \in \mathbb{T}$ de orden infinito. Para cada $\alpha \in \omega_{1} \backslash\{0\}$, definamos $x_{\alpha} \in K$ de la siguiente manera:

$$
x_{\alpha}(\beta)= \begin{cases}a_{\alpha}, & \beta=0 \\ t, & \beta=\alpha ; \\ 0_{\mathbb{T}}, & \text { en otro caso. }\end{cases}
$$

Sea $H$ el subgrupo de $K$ generado por el conjunto $X=\left\{x_{\alpha}: \alpha \in \omega_{1}\right\}$. Mediante un argumento similar al usado en el ejemplo anterior se muestra que $H$ es funcionalmente Hausdorff.

El conjunto $Z=\left(\left\{0_{F}\right\} \times \mathbb{T}^{\omega_{1} \backslash\{0\}}\right) \cap H$ es un conjunto $G_{\delta}$ en $H$. Si $n_{1} x_{\alpha_{1}}+$ $n_{2} x_{\alpha_{2}}+\cdots+n_{k} x_{\alpha_{k}}=z \in Z$, entonces $0_{F}=x(0)=n_{1} a_{\alpha_{1}}+n_{2} a_{\alpha_{2}}+\cdots+n_{k} a_{\alpha_{k}}$ y como el conjunto $\left\{a_{\alpha}: \alpha \in \omega_{1}\right\}$ es independiente, tenemos que $n_{i}=0$ para cada $i \leq k$ y, por lo tanto $z=0_{H}$. De lo anterior, se sigue que $H$ tiene pseudocarácter numerable.

El grupo topológico asociado a $K$ es $K^{*}=\mathbb{R} \times \mathbb{T}^{\omega_{1} \backslash\{0\}}$, donde $\mathbb{R}$ y $\mathbb{T}$ tienen la topología usual. Sea $G$ el subgrupo de $K$ generado por el conjunto $\left\{x_{\alpha}: \alpha \in \omega\right\}$. Veamos que $G^{*}$ es denso en $K^{*}$. Sea $U=\prod_{\alpha \in \omega_{1}} U_{\alpha}$ un abierto canónico no vacío en $K^{*}$. Podemos suponer que $\operatorname{supp}(U)=\left\{0, \alpha_{1}, \ldots, \alpha_{k}\right\}$. Como el grupo generado por $\{x\}$ es denso en $\mathbb{T}^{\omega_{1} \backslash\{0\}}$, existe un entero $r$ tal que $r x\left(\alpha_{i}\right)=r x_{0}\left(\alpha_{i}\right) \in U_{\alpha_{i}}$, para cada $i \leq k$. Como $0_{\mathbb{R}}$ es un punto de acumulación de $A$, el subgrupo generado por $A$ es denso en $\mathbb{R}$. Por lo tanto, existen $\beta \in \omega_{1} \backslash \operatorname{supp}(U)$ y $s \in \mathbb{Z}$ tales que $s a_{\beta} \in U_{0}-r a_{0}$. Consideremos el elemento $y=r x_{0}+s x_{\beta}$. Tenemos que $y(0)=r a_{0}+s a_{\beta} \in U_{0}$ y $y\left(\alpha_{i}\right)=$ $r x_{0}\left(\alpha_{i}\right) \in U_{\alpha_{i}}$, para cada $i \leq k$. Esto implica que $y \in U \cap G^{*}$. Se sigue de lo 
anterior que $G^{*}$ es denso en $K^{*}$ y, por lo tanto, $G^{*}$ es denso en $H^{*}$. Como $G^{*}$ es numerable, podemos concluir que $H^{*}$ es separable.

Sea $M$ el subgrupo de $K$ generado por el conjunto $\left\{x_{\alpha}: \alpha \in \omega_{1} \backslash\{0\}\right\}$. En el ejemplo 2.2.22, se prueba que no existe una condensación de $M$ sobre un espacio Hausdorff primero numerable. Como $M$ es subgrupo de $H$, no existe una condensación de $H$ sobre un espacio Hausdorff primero numerable.

En [6, Open Problem 5.2.1], Arhangel'skii y Tkachenko preguntan: Supongamos que un grupo paratopológico Hausdorff (regular, tychonoff) $G$ contiene un subgrupo denso $\tau$-estrecho. ¿Es $G \tau$-estrecho? El siguiente resultado responde este problema.

Proposición 3.1.22. Si un grupo paratopológico $G$ contiene un subgrupo denso $\tau$-estrecho $H$, entonces $G$ es $\tau$-estrecho.

Demostración. Sea $U$ una vecindad de la identidad $e$ en $G$. Escojamos una vecindad abierta $V$ de $e$ en $G$ la cual satisface $V^{2} \subseteq U$. Por hipótesis, existe un subconjunto $A$ de $H$ tal que $A(V \cap H)=H,(V \cap H) A=H$ y $|A| \leq \tau$. Por el corolario 1.2.6, $G=\bar{H} \subseteq V^{-1} H$, en consecuencia, $G=H V \subseteq A V V \subseteq A U$ y $A U=G$. De forma similar de prueba que $U A=G$.

Definición 3.1.23. Sea $G$ un grupo semitopológico con identidade. El índice izquierdo (derecho) de estrechez de $G$, denotado por $\operatorname{In}_{l}(G)$ (respectivamente, I $n_{r}(G)$ ), es el mínimo cardinal $\tau \geq \omega$ tal que para cada $U \in \mathcal{N}(e)$, existe $F \subseteq G$ tal que $F U=G$ (respectivamente, $U F=G$ ) y $|F| \leq \tau$.

Dado un espacio $X$, denotemos por $\pi \chi(X)$ y $\pi w(X)$ al $\pi$-carácter y al $\pi$-peso de $X$, respectivamente. En [26] se demuestra que todo grupo paratopológico $G$ cumple la igualdad $\pi w(G)=\operatorname{In}_{l}(G) \pi \chi(G)$. Recientemente, en [20] Li, Mou y Wang demuestran la desigualdad $d(G) \leq I n_{l}(G) \chi(G)$ para un grupo semitopológico $G$. El siguiente teorema generaliza los resultados mencionados anteriormente.

Teorema 3.1.24. Para cada grupo semitopológico $G$ se satisface la igualdad $\pi w(G)=\operatorname{In}_{l}(G) \pi \chi(G)$.

Demostración. Claramente, $\pi w(G) \geq \pi \chi(G)$ y $\pi w(G) \geq d(G) \geq I n_{l}(G)$, por lo tanto, $\pi w(G) \geq I n_{l}(G) \pi \chi(G)$. Ahora, veamos que $\pi w(G) \leq I n_{l}(G) \pi \chi(G)$. Para ello, primero probemos la desigualdad $d(G) \leq I n_{l}(G) \pi \chi(G)$. Sea $\gamma$ una $\pi$-base local de $e$ en $G$ con $|\gamma|=\pi \chi(G)$. Para cada $V \in \gamma$, existe 
un subconjunto $A_{V} \subseteq G$ tal que $A_{V} V=G$ y $\left|A_{V}\right| \leq I n_{l}(G)$. Sea $A=$ $\bigcup_{V \in \gamma} A_{V}$. Claramente $|A| \leq \operatorname{In}_{l}(G) \pi \chi(G)$. Demostremos que $A^{-1}$ es denso en $G$. Tomemos $U$ un abierto no vacío en $G$. Fijemos $x \in U$; existe $V \in \gamma$ tal que $V \subseteq U x^{-1}$, equivalentemente, $V x \subseteq U$. Por la definición de $A$ tenemos que $x^{-1} \in a V$, para algún $a \in A$. Se sigue de lo anterior que $a^{-1} \in V x \subseteq U$. Como $A^{-1}$ es denso y $\left|A^{-1}\right| \leq \operatorname{In}_{l}(G) \pi \chi(G)$, podemos concluir que $d(G) \leq$ $\operatorname{In}_{l}(G) \pi \chi(G)$.

Tomemos $D \subseteq G$ tal que que $D$ es denso en $G$ y $|D|=d(G)$. Para cada $d \in D$, sea $\mathcal{B}_{d}$ una $\pi$-base local de $d$ en $G$ con $\left|\mathcal{B}_{d}\right|=\pi \chi(G)$, para cada $d \in D$. Hagamos $\mathcal{B}=\bigcup_{d \in D} \mathcal{B}_{d}$. Es claro que $|\mathcal{B}| \leq \pi \chi(G) d(G)$. Por lo probado anteriormente, tenemos que $|\mathcal{B}| \leq \operatorname{In}_{l}(G) \pi \chi(G)$. Afirmamos que $\mathcal{B}$ es una $\pi$-base de $G$. En efecto, sea $U$ un abierto no vacío en $G$. Como $D$ es denso en $G$, existe $d \in D \cap U$. Como $\mathcal{B}_{d}$ es una $\pi$-base local de $d$ en $G$ podemos encontrar $V \in \mathcal{B}_{d} \subseteq \mathcal{B}$ tal que $V \subseteq U$. Se sigue de lo anterior que $\pi w(G)=\operatorname{In}_{l}(G) \pi \chi(G)$.

Corolario 3.1.25. Todo grupo semitopológico $\omega$-estrecho con $\pi$-carácter numerable tiene una $\pi$-base numerable.

Corolario 3.1.26. Todo grupo semitopológico $\omega$-estrecho con $\pi$-carácter numerable es separable.

En [6, Open problem 5.2.3] se plantea la siguiente cuestion:

Sea $G$ un grupo paratopológico Hausdorff $\tau$-estrecho tal que $\psi(G) \leq \tau$. ¿Es la cardinalidad de $G$ menor o igual que $2^{\tau}$ ?

En [29, Theorem 2.28] M. Sanchis y M. Tkachenko demuestran que todo grupo paratopológico Hausdorff Lindelöf de pseudocarácter numerable $G$ satisface $|G| \leq 2^{\omega}$. En realidad ellos demuestran lo siguiente:

Proposición 3.1.27. Todo grupo semitopológico Hausdorff G cumple

$$
|G| \leq 2^{H s(G) \psi(G) I n_{r}(G)} .
$$

Demostración. Sea $\kappa=H s(G) \psi(G) \operatorname{In}_{r}(G)$. Podemos encontrar una familia $\Gamma \subseteq \mathcal{N}(e)$ tal que $\bigcap_{V \in \Gamma} V V^{-1}=\{e\}$ y $|\Gamma| \leq \kappa$. Para cada $V \in \Gamma$, existe un subconjunto $A_{V} \subseteq G$ tal que $V A_{V}=G$ y $\left|A_{V}\right| \leq \kappa$. Consideremos la familia $\mathcal{P}=\left\{V a: V \in \Gamma, a \in A_{V}\right\}$. Veamos que $\mathcal{P}$ es una subbase para una topología $T_{1}$ en $G$. Tomemos $x, y \in G$ con $y \neq x$; existe $V \in \Gamma$ tal que $x y^{-1} \notin V V^{-1}$. Escojamos $a \in A_{V}$ tal que $x \in V a$. Si $y \in V a$, entonces 
$x y^{-1} \in V a a^{-1} V^{-1}=V V^{-1}$, lo cual contradice la elección de $V$. Si $\tau$ es la topología generada por $\mathcal{P}$ en $G$, entonces $(G, \tau)$ es un espacio $T_{1}$ de peso menor o igual que $\kappa$. Aplicando $[17,2.2]$, tenemos que $|G| \leq 2^{\kappa}$.

\subsection{El rango de la diagonal}

Los siguientes tres lemas son útiles en la demostración del teorema 3.2.4.

Lema 3.2.1. Si $G$ es un grupo semitopológico Hausdorff con $\pi$-carácter numerable, entonces $G$ tiene pseudocarácter numerable.

Demostración. Sea $\gamma=\left\{U_{n}: n \in \omega\right\}$ una $\pi$-base local de la identidad $e$ en $G$. Hagamos $W_{n}=U_{n} U_{n}^{-1}$ para cada $n \in \omega$. Afirmamos que $\bigcap_{n \in \omega} W_{n}=\{e\}$. En efecto, sea $x$ un elemento de $G$ distinto de la identidad. Como $G$ es Hausdorff, existe una vecindad abierta $V$ de $e$ tal que $x V \cap V=\emptyset$. Como $\gamma$ es una $\pi$-base local de $e$, existe $n \in \omega$ tal que $U_{n} \subseteq V$. En consecuencia, $x U_{n} \cap U_{n}=\emptyset$, es decir, $x \notin U_{n} U_{n}^{-1}=W_{n}$.

Lema 3.2.2. Sean $G$ un grupo semitopológico, $A$ un subconjunto de $G$ y $\gamma$ una $\pi$-base local de la identidad e en $G$. Entonces,

$$
\bigcap_{V \in \gamma} A V^{-1} \subseteq \bar{A}
$$

Demostración. Denotemos por $\mathcal{N}(e)$ a la familia de vecindades abiertas de e. Sabemos que $\bigcap_{U \in \mathcal{N}(e)} A U^{-1}=\bar{A}$. Si la intersección $\bigcap_{V \in \gamma} A V^{-1}$ es vacía, terminamos. En otro caso, tomemos $x \in \bigcap_{V \in \gamma} A V^{-1}$. Veamos que $x \in$ $\bigcap_{U \in \mathcal{N}(e)} A U^{-1}$. Tomemos $U \in \mathcal{N}(e)$. Como $\gamma$ es una $\pi$-base local de $e$, existe $V \in \gamma$ tal que $V \subseteq U$, en consecuencia $x \in A V^{-1} \subseteq A U^{-1}$. Hemos demostrado que $\bigcap_{V \in \gamma} A V^{-1} \subseteq \bigcap_{U \in \mathcal{N}(e)} A U^{-1}=\bar{A}$.

Dada una colección $\mathcal{U}$ de subconjuntos de un espacio $X$, se define la estrella de $\mathcal{U}$ respecto a un conjunto $A \subseteq X$ como $\operatorname{st}(\mathcal{U}, A)=\bigcup\{U: U \in$ $\mathcal{U}, U \cap A \neq \emptyset\}$. Cuando $A=\{x\}$, escribimos $s t(\mathcal{U}, x)$. Hagamos $s t^{1}(\mathcal{U}, x)=$ $s t(\mathcal{U}, x)$ y definimos recursivamente $s t^{n+1}(\mathcal{U}, x)=s t\left(\mathcal{U}, s t^{n}(\mathcal{U}, x)\right)$.

Decimos que un espacio $X$ tiene diagonal $G_{\delta}$ de rango $k \in \mathbb{N}$ si existe una sucesión $\left\{\mathcal{U}_{n}: n \in \omega\right\}$ de cubiertas abiertas de $X$ tal que $\bigcap\left\{s t^{k}\left(\mathcal{U}_{n}, x\right)\right.$ : $n \in \omega\}=\{x\}$, para cada $x \in X$. Si un espacio tiene diagonal $G_{\delta}$ de rango $k$ para todo número natural $k$, decimos que $X$ tiene una diagonal $G_{\delta}$ de rango infinito. 
Lema 3.2.3. Sea $G$ un grupo paratopológico con identidad e. Si existe una familia $\left\{W_{n}: n \in \omega\right\} \subseteq \mathcal{N}(e)$ tal que para cada par de puntos distintos $y, z \in G$ podemos encontrar $n \in \omega$ el cual satisface $W_{n} y \cap z W_{n}=\emptyset$, entonces $G$ tiene diagonal de rango infinito.

Demostración. Sea $k$ un número natural arbitrario. Veamos que $G$ tiene una diagonal $G_{\delta}$ de rango $k$. Como $G$ es un grupo paratopológico, podemos construir una sucesión $\left\{V_{n}: n \in \omega\right\} \subseteq \mathcal{N}(e)$ tal que $V_{n}^{k} \subseteq W_{n}$ para cada $n \in \omega$. Sea $U(x, n)=V_{n} x \cap x V_{n}$ para cada $n \in \omega$ y $x \in G$. Haga$\operatorname{mos} \beta_{n}=\{U(x, n): x \in G\}$ para cada $n \in \omega$. Consideremos la familia $\left\{\beta_{n}: n \in \omega\right\}$ de cubiertas abiertas de $G$. Fijemos $y \in G$. Probemos que $\bigcap\left\{s t^{k}\left(\beta_{n}, y\right): n \in \omega\right\}=\{y\}$. Sea $z \in G$ con $z \neq y$. Por hipótesis, existen $n \in \omega$ tal que $W_{n} y \cap z W_{n}=\emptyset$. Afirmamos que $z \notin s t^{k}\left(\beta_{n}, y\right)$. Supongamos lo contrario; entonces existen $x_{1}, \ldots, x_{k} \in G$ tales que $y \in U\left(x_{1}, n\right)$, $z \in U\left(x_{k}, n\right)$ y $U\left(x_{i}, n\right) \cap U\left(x_{i+1}, n\right) \neq \emptyset$ para $1 \leq i \leq k-1$. En consecuencia, existen puntos $p_{1}, \ldots, p_{k}, q_{0}, \ldots, q_{k-1} \in V_{n}$, los cuales satisfacen $y=x_{1} q_{0}, z=p_{k} x_{k}$ y $p_{i} x_{i}=x_{i+1} q_{i}$ (equivalentemente, $x_{i}=p_{i}^{-1} x_{i+1} q_{i}$ ) para $1 \leq i \leq k-1$. De lo anterior podemos concluir:

$$
\begin{aligned}
& y=x_{1} q_{0}=p_{1}^{-1} x_{2} q_{1} q_{0}=\cdots=p_{1}^{-1} p_{2}^{-1} \cdots p_{k-1}^{-1} x_{k} q_{k-1} \cdots q_{1} q_{0}= \\
& p_{1}^{-1} p_{2}^{-1} \cdots p_{k-1}^{-1} p_{k}^{-1} z q_{k-1} \cdots q_{0} \in V_{n}^{-k} z V_{n}^{k} \subseteq W_{n}^{-1} z W_{n} .
\end{aligned}
$$

Lo anterior implica que $W_{n} y \cap z W_{n} \neq \emptyset$, lo cual contradice la elección de $W_{n}$. Por lo tanto, $G$ tiene una diagonal $G_{\delta}$ de rango $k$ para todo $k \in \mathbb{N}$. El lema queda demostrado.

En [2, Problem 3], se plantea la siguiente pregunta:

Sea $G$ un grupo paratopológico Hausdorff (regular) con $\pi$-carácter numerable. ¿Tiene $G$ una diagonal $G_{\delta}$ de rango infinito?

El siguiente resultado responde en forma positiva a esta pregunta en el caso de grupos paratopológicos regulares.

Teorema 3.2.4. Si $G$ es un grupo paratopológico regular con $\pi$-carácter numerable, entonces $G$ tiene una diagonal $G_{\delta}$ de rango infinito.

Demostración. Por el lema 3.2.1, G tiene pseudocarácter numerable. Además, como $G$ es un grupo paratopológico regular, podemos construir una sucesión $\left\{V_{n}: n \in \omega\right\} \subseteq \mathcal{N}(e)$ tal que $\bigcap_{n \in \omega} V_{n}=\{e\}$ y $\overline{V_{n+1} V_{n+1}} \subseteq V_{n}$ para cada $n \in \omega$. Sea $\left\{U_{n}: n \in \omega\right\}$ una $\pi$-base local de $e$. Hagamos $W_{n}=U_{n} U_{n}^{-1}$ para cada $n \in \omega$. 
Tomemos un par de puntos distintos $y, z \in G$. Por la definición de la sucesión $\left\{V_{n}: n \in \omega\right\}$, existe $n \in \omega$ tal que $y z^{-1} \notin \overline{V_{n} V_{n}}$, equivalentemente, $y \notin \overline{V_{n} V_{n}} z$. Podemos encontrar $W \in \mathcal{N}(e)$ tal que $z W \subseteq V_{n} z$. Observemos que la familia $\left\{U_{m}: U_{m} \subseteq W\right\}$ es una $\pi$-base local de $e$. Por el lema 3.2.2, tenemos que $\bigcap\left\{V_{n} z W U_{m}^{-1}: U_{m} \subseteq W\right\} \subseteq \overline{V_{n} z W}$. De lo anterior, podemos concluir:

$$
\overline{V_{n} V_{n}} z=\overline{V_{n} V_{n} z} \supseteq \overline{V_{n} z W} \supseteq \bigcap\left\{V_{n} z W U_{m}^{-1}: U_{m} \subseteq W\right\} .
$$

Como $y \notin \overline{V_{n} V_{n}} z$, vemos que $y \notin \bigcap\left\{V_{n} z W U_{m}^{-1}: U_{m} \subseteq W\right\}$, es decir, existe $m \in \omega$ tal que $U_{m} \subseteq W$ y $y \notin V_{n} z W U_{m}^{-1}$. Como $U_{m} \subseteq W, y \notin V_{n} z U_{m} U_{m}^{-1}=$ $V_{n} z W_{m}$, equivalentemente, $y W_{m}^{-1} \cap V_{n} z=\emptyset$. Sabemos que $W_{m}=W_{m}^{-1}$, por lo tanto, $y W_{m} \cap V_{n} z=\emptyset$. Sea $O_{n, m}=V_{n} \cap W_{m}$ para cada $n, m \in \omega$. Observemos que la familia $\left\{O_{n, m}: n, m \in \omega\right\}$ satisface las condiciones del lema 3.2.3, por lo tanto, $G$ tiene una diagonal $G_{\delta}$ de rango infinito.

El siguiente lema es bien conocido, así que omitimos su demostración.

Lema 3.2.5. Si $Y$ es un subespacio denso de un espacio regular $X$, entonces $\pi \chi(y, Y)=\pi \chi(y, X)$ (respectivamente, $\chi(y, Y)=\chi(y, X))$ para cada $y \in Y$.

Sea $(G, \tau)$ un grupo paratopológico. Consideremos la regularización $r(G)=$ $\left(G, \tau_{r}\right)$ de $G$ (ver sección 1.4). Dado un subgrupo $K$ de $G$, hagamos $\sigma=\left.\tau\right|_{K}$. En general, las topologías $\left.\tau_{r}\right|_{K}$ y $\sigma_{r}$ no coinciden. En el siguiente resultado damos condiciones suficiente para que dichas topologías coincidan.

Lema 3.2.6. Sea $H$ un subgrupo denso de un grupo paratopológico $(G, \tau)$. Si $\sigma=\left.\tau\right|_{H}$, entonces $\left.\tau_{r}\right|_{H}=\sigma_{r}$.

Demostración. Sea $\mathcal{B}$ una base local de la identidad $e$ en $(G, \tau)$. La familia $\mathcal{B}_{\sigma_{r}}=\left\{\right.$ int $\left._{\sigma}\left(\overline{U \cap H^{\sigma}}\right): U \in \mathcal{B}\right\}$ es una base local de $e$ en $\left(H, \sigma_{r}\right)$. Por otro lado, la familia $\mathcal{B}_{\left.\tau_{r}\right|_{H}}=\left\{\left(\right.\right.$ int $\left.\left._{\tau} \bar{U}^{\tau}\right) \cap H: U \in \mathcal{B}\right\}$ es una base local de $\left(H,\left.\tau_{r}\right|_{H}\right)$. Probemos que $\mathcal{B}_{\sigma_{r}}=\mathcal{B}_{\left.\tau_{r}\right|_{H}}$. Dado $U \in \mathcal{B}$ tenemos int $\sigma\left(\overline{U \cap H^{\sigma}}\right)=$ int $_{\sigma}\left[\left(\overline{U \cap H}^{\tau}\right) \cap H\right]=\operatorname{int}_{\sigma}\left(\bar{U}^{\tau} \cap H\right)$. El conjunto $\left(\right.$ int $\left._{\tau} \bar{U}^{\tau}\right) \cap H$ es un abierto en $(H, \sigma)$ que está contenido en $\bar{U}^{\tau} \cap H$, por lo tanto, $\left(\right.$ int $\left._{\tau} \bar{U}^{\tau}\right) \cap H \subseteq$ int $_{\sigma}\left(\bar{U}^{\tau} \cap\right.$ $H)$. Para obtener la otra contención, observemos que int $\sigma_{\sigma}\left(\bar{U}^{\tau} \cap H\right) \subseteq H$. Si $x \in$ int $_{\sigma}\left(\bar{U}^{\tau} \cap H\right)$, entonces existe $V \in \tau$ tal que $x \in V \cap H \subseteq \bar{U}^{\tau} \cap H \subseteq \bar{U}^{\tau}$,

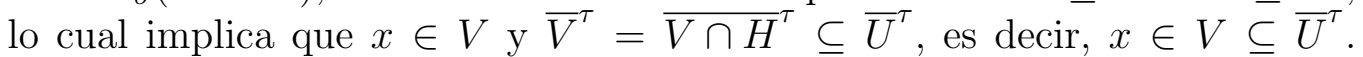
En consecuencia $x \in \operatorname{int}_{\tau} \bar{U}^{\tau}$. De lo anterior se sigue que $\operatorname{int}_{\sigma}\left(\overline{U \cap \bar{H}^{\sigma}}\right)=$ $\left(\right.$ int $\left._{\tau} \bar{U}^{\tau}\right) \cap H$ para cada $U \in \mathcal{B}$. 
Proposición 3.2.7. Sea $H$ un subgrupo denso de un grupo paratopológico Hausdorff $G$ tal que $H$ tiene $\pi$-carácter numerable (carácter numerable). Entonces, la regularización $r(G)$ de $G$ tiene $\pi$-carácter numerable (carácter numerable).

Demostración. Hagamos $\sigma=\left.\tau\right|_{H}$. Como $(H, \sigma)$ tiene $\pi$-carácter numerable (carácter numerable), el grupo $\left(H, \sigma_{r}\right)$ tiene $\pi$-carácter numerable (carácter numerable). Por el lema 3.2.6, $\left(H,\left.\tau_{r}\right|_{H}\right)$ tiene $\pi$-carácter numerable (carácter numerable). Además, el grupo paratopológico $\left(G, \tau_{r}\right)$ es regular. Aplicando el lema 3.2.5, podemos concluir que $\left(G, \tau_{r}\right)$ tiene $\pi$-carácter numerable (carácter numerable).

En el siguiente resultado respondemos la pregunta planteada por A. Arhangel'skii y A. Bella para el caso Hausdorff (ver [2, Problem 3]), en una forma más general (ver ejemplo 3.2.9).

Teorema 3.2.8. Sea $H$ un subgrupo denso de un grupo paratopológico Hausdorff $G$ tal que $H$ tiene $\pi$-carácter numerable. Entonces, $G$ tiene una diagonal $G_{\delta}$ de rango infinito. En particular, todo grupo paratopológico Hausdorff con $\pi$-carácter numerable tiene una diagonal $G_{\delta}$ de rango infinito.

Demostración. Por la proposición 3.2.7, la regularización $r(G)$ de $G$ es un grupo paratopológico regular con $\pi$-carácter numerable. Aplicando el teorema 3.2.4, podemos concluir que el espacio $\left(G, \tau_{r}\right)$ tiene diagonal $G_{\delta}$ de rango infinito. Como $\tau_{r} \subseteq \tau, G$ tiene diagonal $G_{\delta}$ de rango infinito.

Es sabido que si un grupo topológico Hausdorff $G$ contiene un subgrupo denso primero numerable, entonces $G$ es primero numerable. El siguiente ejemplo se muestra que este hecho no es válido para grupos paratopológcos.

Ejemplo 3.2.9. Existe un grupo paratopológico funcionalmente Hausdorff $G$ de $\pi$-carácter no numerable el cual contiene a un subgrupo denso segundo numerable.

Demostración. Sea $F=(\mathbb{R}, \mu)$ el grupo paratopológico que tiene como base local del cero a la familia $\left\{U_{n}=\left(-\frac{1}{n}, \frac{1}{n}\right) \cup(n, \infty): n \in \mathbb{N}\right\}$. El espacio $F$ es $T_{1}$ segundo numerable (ver ejemplo 2.2.16). Consideremos el grupo del círculo $\mathbb{T}$ con notación aditiva y su topología usual.

Tomemos un conjunto $S=\left\{t_{\alpha}: \alpha \in \omega_{1} \backslash\{0\}\right\} \subseteq \mathbb{T}$ linealmente independiente, donde $t_{\alpha}$ es de orden infinito para cada $\alpha \in \omega_{1} \backslash\{0\}$. Sea 
$K=\mathbb{T} \times F^{\omega_{1} \backslash\{0\}}$. Para cada $\alpha \in \omega_{1} \backslash\{0\}$, definamos $x_{\alpha}$ de la siguiente manera:

$$
x_{\alpha}(\beta)= \begin{cases}t_{\alpha}, & \beta=0 \\ -1, & \beta=\alpha \\ 1, & \text { en otro caso. }\end{cases}
$$

Denotemos por $G$ y $H$ a los subgrupos de $K$ generados por los conjuntos $X=\left\{x_{\alpha}: \alpha \in \omega_{1} \backslash\{0\}\right\}$ y $\left\{x_{n}: n \in \omega \backslash\{0\}\right\}$, respectivamente. Es claro que $H$ es segundo numerable. Veamos que $G$ es funcionalmente Hausdorff. Sea $M$ el subgrupo de $\mathbb{T}$ generado por $S$. Denotemos por $p$ a la proyección de $K$ sobre $\mathbb{T}$. Entonces $\left.p\right|_{G}$ es un isomorfismo continuo de $H$ sobre $M$. Debido a que $M$ es completamente regular, podemos concluir que $G$ es funcionalmente Hausdorff.

Probemos que $H$ es denso en $G$. Para ello veamos que $x_{\alpha},-x_{\alpha} \in \bar{H}^{G}$ para cada $\alpha \in \omega_{1} \backslash \omega$. Sea $O=\prod\left\{O_{\alpha}: \alpha \in \omega_{1}\right\}$ un abierto canónico no vacío en $K$ tal que $0_{K} \in O$. Hagamos $\operatorname{supp}(O)=\left\{0, \alpha_{1}, \alpha_{2}, \ldots, \alpha_{k}\right\}$ y $V=O \cap G$. Tomemos $n \in \mathbb{N} \backslash \operatorname{supp}(O)$. Por la topología en los espacios $\mathbb{T}$ y $F$, podemos encontrar un entero positivo $m>2$ tal que $m t_{n} \in\left(O_{0}-t_{\alpha}\right)$ y $(m-1) \in O_{\alpha_{i}}$ para cada $0<i \leq k$. Si $y=m x_{n}+x_{\alpha}$, entonces

$$
\begin{aligned}
& y(0)=m t_{n}+t_{\alpha} \in O_{0} . \text { Además, } \\
& y\left(\alpha_{i}\right)=m \pm 1 \in O_{\alpha_{i}} \text { si } 0<i \leq k .
\end{aligned}
$$

De lo anterior podemos concluir que $y \in V$, lo cual implica que $\left(-x_{\alpha}+V\right) \cap$ $H \neq \emptyset$, así que $-x_{\alpha} \in \bar{H}^{G}$. La demostración de que $x_{\alpha} \in \bar{H}^{G}$ es similar, sólo basta tomar un entero positivo $r$ tal que $r t_{n} \in\left(O_{0}+t_{\alpha}\right)$ y $r-1 \in O_{\alpha_{i}}$ para cada $0<i \leq k$ y hacer $y=r x_{n}-x_{\alpha}$. Como $\bar{H}^{G}$ es un subsemigrupo de $G$ que contiene a $X \cup(-X)$ y el grupo $G$ está generado por el conjunto $X$, tenemos que $\bar{H}^{G}=G$.

Por último, probemos que $G$ no tiene $\pi$-carácter numerable. Supongamos lo contrario. Sea $\left\{V_{n}: n \in \omega\right\}$ una $\pi$-base local de $0_{G}$ en $G$. Para cada $n \in \omega$ tomemos un abierto canónico no vacío $W_{n}$ en $K$ tal que $W_{n} \cap G \subseteq V_{n}$. Sea $P=\{0\} \cup \bigcup\left\{\operatorname{supp}\left(W_{n}\right): n \in \omega\right\}$. Fijemos $\alpha \in \omega_{1} \backslash S$. Consideremos el abierto canónico $W \in \mathcal{N}_{K}(0)$ tal que $\operatorname{supp}(W)=\alpha$ y $W_{\alpha}=U_{1} \subseteq F$. Por hipótesis, existe $n \in \omega$ tal que $V_{n} \subseteq W \cap G$. Podemos encontrar un entero positivo $m$ tal que $m x_{\alpha} \in W_{n} \cap G \subseteq V_{n}$. Sin embargo, $m x_{\alpha} \notin W$, lo cual es una contradicción, así podemos concluir que $G$ no tiene $\pi$-carácter numerable. 
De acuerdo a [2, Proposition 1], todo espacio con una diagonal $G_{\delta}$ de rango 3 tiene una diagonal $G_{\delta}$ regular.

Corolario 3.2.10. Cada grupo paratopológico Hausdorff con $\pi$-carácter numerable tiene una diagonal $G_{\delta}$ regular.

Un subconjunto $B$ de un espacio $X$ se dice acotado en $X$ si cada familia infinita $\xi$ de subconjuntos abiertos de $X$ tal que $V \cap B \neq \emptyset$, para cada $V \in \xi$, tiene un punto de acumulación en $X$. Si $X$ es acotado en él mismo, entonces decimos que $X$ es débilmente compacto. Se puede demostrar que en espacios Tychonoff, compacidad débil es equivalente a pseudocompacidad.

En [3, Theorem 1] se prueba que si $X$ es un espacio regular con una diagonal $G_{\delta}$ regular, entonces la cerradura de cada subconjunto acotado $B$ de $X$ es metrizable. Este resultado junto con el teorema 3.2.10 nos permiten establecer los siguientes dos corolarios.

Corolario 3.2.11. Sea $G$ un grupo paratopológico regular con $\pi$-carácter numerable. Entonces cada subconjunto acotado en $G$ es metrizable.

Corolario 3.2.12. Si $G$ es un grupo paratopológico regular con $\pi$-carácter numerable, entonces cada subespacio débilmente compacto de $G$ es compacto y metrizable.

Demostración. Si $B$ un subespacio débilmente compacto de $G$, entonces $B$ es metrizable y pseudocompacto. Además, cada espacio pseudocompacto metrizable es compacto. Esto termina la prueba.

Es inmediato de la definición de índice de regularidad (ver sección 3.1), que todo grupo semitopológico regular primero numerable tiene índice de regularidad numerable. En el siguiente resultado mejoramos este hecho para el ámbito de grupos paratopológicos.

Proposición 3.2.13. Si $G$ es un grupo paratopológico regular, entonces $\operatorname{Ir}(G) \leq \pi \chi(G)$.

Demostración. Sea $U \in \mathcal{N}(e)$. Por la regularidad de $G$, existe $V \in \mathcal{N}(e)$ tal que $\overline{V V} \subseteq U$. Sea $\gamma$ una $\pi$-base local de $e$ tal que $|\gamma|=\pi \chi(G)$. Hagamos $\lambda=\{W \in \gamma: W \subseteq V\}$. Es claro que $\lambda$ es una $\pi$-base local de $e$ con $|\lambda|=$ $\pi \chi(G)$. Por el lema 1.2.5, $\bigcap_{W \in \lambda} V\left(W V^{-1}\right)^{-1}=\bigcap_{W \in \lambda} V V W^{-1} \subseteq \overline{V V} \subseteq U$. Como $W \subseteq V$ para cada $W \in \lambda$, el conjunto $W V^{-1}$ es una vecindad abierta de $e$ para cada $W \in \lambda$. Se sigue de lo anterior que $\operatorname{Ir}(G) \leq \pi \chi(G)=|\lambda|$. 
El número de Hausdorff de un grupo semitopológico Hausdorff $G$, denotado por $H s(G)$, es el mínimo cardinal $\kappa$ tal que para cada vecindad $U$ de $e$ en $G$, podemos encontrar una familia $\gamma \subseteq \mathcal{N}(e)$ tal que $\bigcap_{V \in \gamma} V V^{-1} \subseteq U$ y $|\gamma| \leq \kappa$ (ver [32]). Se sigue de la definición que todo grupo semitopológico Hausdorff primero numerable tiene número de Hausdorff numerable.

Proposición 3.2.14. Todo grupo paratopológico Hausdorff satisface la desigualdad $H s(G) \leq \pi \chi(G)$.

Demostración. Sea $\gamma$ una $\pi$-base local de $e$ tal que $|\gamma|=\pi \chi(G)$. Como $G$ es un grupo paratopológico Hausdorff, para cada $x \neq e$, existe una vecindad de la identidad $V_{x}$ tal que $V_{x} x V_{x} \cap V_{x}^{2}=\emptyset$, equivalentemente, $x \notin$ $\left(V_{x}^{-1} V_{x}\right)\left(V_{x} V_{x}^{-1}\right)$. Podemos encontrar $U \in \gamma$ tal que $U \subseteq V_{x}$ y por lo tanto, $x \notin\left(U^{-1} U\right)\left(U U^{-1}\right)$. Sea $W_{U}=\left(U^{-1} U\right) \cap\left(U U^{-1}\right)$ para cada $U \in \gamma$. Se sigue de lo anterior que $\bigcap\left\{W_{U} W_{U}^{-1}: U \in \gamma\right\}=\{e\}$.

En un intento de generalizar a grupos semitopológicos las dos proposiciones anteriores, se plantea la siguiente cuestión:

Problema 3.2.15. Sea $G$ un grupo semitopológico regular con $\pi$-carácter numerable. ¿Tiene $G$ índice de regularidad (número de Hausdorff) numerable?

Un grupo semitopológico se dice $\omega$-balanceado si para cada vecindad $U$ de la identidad $e$ en $G$, existe una familia numerable $\gamma$ tal que para cada $x \in G$ podemos encontrar un elemento $V \in \gamma$ el cual satisface $x V x^{-1} \subseteq U$.

Proposición 3.2.16. Si $G$ es un grupo paratopológico Hausdorff $\omega$-balanceado con $H s(G) \psi(G) \leq \omega$, entonces $G$ tiene una diagonal $G_{\delta}$ de rango infinito.

Demostración. Como $H s(G) \psi(G) \leq \omega$, existe una familia $\left\{U_{n}: n \in \omega\right\} \subseteq$ $\mathcal{N}_{G}(e)$ tal que $\bigcap_{n \in \omega} U_{n} U_{n}^{-1}=\{e\}$, donde $e$ es la identidad de $G$. Como $G$ es $\omega$-balanceado, para cada $n \in \omega$, existe una familia numerable $\gamma_{n}$ tal que para cada $x \in G$ podemos encontrar $V \in \gamma_{n}$ tal que $x^{-1} V x \subseteq U_{n}$. Sea $\gamma=\bigcup_{n \in \omega} \gamma_{n}$. Veamos que la familia numerable $\gamma \cup\left\{U_{n}: n \in \omega\right\}$ satisface las condiciones del lema 3.2.3. Tomemos una pareja de puntos distintos $x, y \in G$. Como $\bigcap_{n \in \omega} U_{n} U_{n}^{-1}=\{e\}$, existe $n \in \omega$ tal que $x^{-1} y \notin U_{n} U_{n}^{-1}$, equivalentemente, $x U_{n} \cap y U_{n}=\emptyset$. Por la construcción de la familia $\gamma$, existe $V \in \gamma$ tal que $V x \subseteq x U_{n}$. En consecuencia $V x \cap y U_{n}=\emptyset$. Aplicando el lema 3.2 .3 , concluimos que $G$ tiene una diagonal $G_{\delta}$ de rango infinito. 
Es claro que todo grupo semitopológico primero numerable es $\omega$-balanceado. Esto motiva la siguiente pregunta:

Problema 3.2.17. ¿Todo grupo paratopológico Hausdorff (regular) con $\pi$ carácter numerable es $\omega$-balanceado?

\subsection{Condensaciones}

Decimos que un espacio $X$ se puede condensar sobre un espacio $Y$ si existe una función continua y biyectiva de $X$ en $Y$.

Teorema 3.3.1. Sea $G$ un grupo semitopológico completamente regular $\omega$ estrecho tal que $H s(G) \psi(G) \leq \omega$. Entonces $G$ se puede condensar sobre un espacio metrizable segundo numerable.

Demostración. Por hipótesis, existe una familia $\left\{W_{n}: n \in \omega\right\} \subseteq \mathcal{N}(e)$ la cual satisface $\bigcap_{n \in \omega} W_{n} W_{n}^{-1}=\{e\}$. Como $G$ es completamente regular, para cada $n \in \omega$, existe una función continua $f_{n}: G \rightarrow[0,1]$ tal que $f_{n}(e)=1$ y $f_{n}\left(G \backslash W_{n}\right) \subseteq\{0\}$. Hagamos $V_{n}=\left\{x \in G: f_{n}(x) \neq 0\right\}$. Claramente $V_{n}$ es una vecindad abierta de la identidad contenida en $W_{n}$ para cada $n \in \omega$, así que $\bigcap_{n \in \omega} V_{n} V_{n}^{-1}=\{e\}$. Como $G$ es $\omega$-estrecho, para cada $n \in \omega$, existe $A_{n} \subseteq G$ numerable tal que $V_{n} A_{n}=G$. Sea $\mathcal{P}=\left\{V_{n} a: n \in \omega, a \in A_{n}\right\}$. Claramente, la familia $\mathcal{P}$ es numerable. Para cada $n \in \omega$ y $a \in A_{n}$ definamos la función continua $f_{n, a}: G \rightarrow[0,1]$ como $f_{n, a}=f_{n} \circ \rho_{a^{-1}}$. Aquí, $\rho_{a^{-1}}$ es la traslación derecha de $G$ por $a^{-1}$. Observemos que $f_{n, a}\left(V_{n} a\right)=f_{n} \circ \rho_{a^{-1}}\left(V_{n} a\right)=f_{n}\left(V_{n}\right) \subseteq$ $(0,1]$. Si $x \notin V_{n} a$, entonces $\rho_{a^{-1}}(x) \notin V_{n}$, en consecuencia $f_{n, a}(x)=0$. Se sigue de lo anterior que $V_{n} a=\left\{x \in G: f_{n, a}(x) \neq 0\right\}$. Veamos que la familia de funciones continuas $\mathcal{F}=\left\{f_{n, a}: n \in \omega, a \in A_{n}\right\}$ separa a los puntos de $G$. Tomemos un par de puntos distintos $x, y \in G$; existe $n \in \omega$ tal que $x y^{-1} \notin V_{n} V_{n}^{-1}$. Escojamos $a \in A_{n}$ tal que $x \in V_{n} a$. Si $y \in V_{n} a$, entonces $x y^{-1} \in V_{n} a a^{-1} V_{n}^{-1}=V_{n} V_{n}^{-1}$, lo cual contradice la elección de $V_{n}$. Por lo tanto, $x \in V_{n} a$ y $y \notin V_{n} a$. De lo anterior, podemos concluir que $f_{n, a}(x) \neq 0$ y $f_{n, a}(y)=0$. Como la familia de funciones continuas $\mathcal{F}$ separa puntos y es numerable, tenemos que el producto diagonal $\triangle_{f \in \mathcal{F}} f: G \rightarrow[0,1]^{\omega}$ es una función continua inyectiva. El teorema queda demostrado.

En [33] M. Tkachenko plantea el problema: ¿cada grupo paratopológico Hausdorff (o regular) $G \operatorname{con} l(G) \psi(G) \leq \omega$ se puede condensar sobre 
un espacio Hausdorff segundo numerable? A continuación se responde esta pregunta.

Teorema 3.3.2. Si $G$ es un grupo paratopológico regular con $l(G) \psi(G) \leq \omega$, entonces $G$ se puede condensar sobre un espacio métrico segundo numerable.

Demostración. Como $G$ es regular Lindelöf, el espacio $G$ es completamente regular. También, todo grupo paratopológico Hausdorff Lindelöf es $\omega$ estrecho y tiene número de Hausdorff numerable (ver [32, Proposition 2.4]). Por el teorema anterior, $G$ se puede condensar sobre un espacio métrico separable.

Utilizando la regularización de grupo paratopológico podemos extender el teorema 3.3.2 a grupos paratopológicos Hausdorff como se muestra en el teorema 3.3.4. Para ello, necesitamos probar el siguiente resultado.

Proposición 3.3.3. Si $G$ es un grupo paratopológico Hausdorff que satisface $H s(G) \psi(G) \leq \omega$. Entonces la regularización $r(G)$ de $G$ también tiene número de Hausdorff y pseudocarácter numerable.

Demostración. El grupo paratopológico $G$ satisface $H s(G) \psi(G) \leq \omega$ si y sólo si existe una familia $\left\{V_{n}: n \in \omega\right\} \subseteq \mathcal{N}_{G}(e)$ tal que $\bigcap_{n \in \omega} V_{n} V_{n}^{-1}=\{e\}$, donde $e$ es la identidad de $G$. Sea $W_{n}=\operatorname{Int} \bar{V}_{n}$, para cada $n \in \omega$. Cada $W_{n}$ es una vecindad abierta de la identidad en $r(G)$. Tomemos $x \in G$ con $x \neq e$. Como $\bigcap_{n \in \omega} V_{n} V_{n}^{-1}=\{e\}$, existe $n \in \omega$ tal que $x V_{n} \cap V_{n}=\emptyset$. En consecuencia, $x \operatorname{Int} \bar{V}_{n} \cap \operatorname{Int} \bar{V}_{n}=\emptyset$, equivalentemente, $x \notin W_{n} W_{n}^{-1}$. Se sigue de lo anterior que la familia $\left\{W_{n}: n \in \omega\right\}$ satisface $\bigcap_{n \in \omega} W_{n} W_{n}^{-1}=\{e\}$. Por lo tanto, $r(G)$ tiene número de Hausdorff y pseudocarácter numerable.

Teorema 3.3.4. Si $G$ es un grupo paratopológico Hausdorff con $l(G) \psi(G) \leq$ $\omega$, entonces $G$ se puede condensar sobre un espacio métrico segundo numerable.

Demostración. Todo grupo paratopológico Hausdorff Lindelöf tiene número de Hausdorff numerable (ver [32, Proposition 2.4]). Aplicando la proposición anterior, tenemos que el grupo paratopológico $r(G)$ tiene número de Hausdorff y pseudocarácter numerable. Para finalizar la demostración basta aplicar el teorema 3.3.2 a la regularización $r(G)$ de $G$.

El siguiente hecho es fácil de probar, así que omitimos su demostración. 
Proposición 3.3.5. Todo espacio métrico tiene diagonal $G_{\delta}$ de rango infinito.

Corolario 3.3.6. Todo grupo paratopológico Hausdorff Lindelöf con pseudocarácter numerable tiene diagonal $G_{\delta}$ de rango infinito.

Demostración. Sea $(G, \tau)$ un grupo paratopológico el cual satisface las condiciones del enunciado. Por el teorema 3.3.4, exite una topología $\sigma \subseteq \tau$ en $G$ tal que $X=(G, \sigma)$ es un espacio métrico. Aplicando la proposición 3.3.5, concluimos que $X$ tiene una diagonal $G_{\delta}$ de rango infinito, es decir, para cada número natural $k$ existe una sucesión $\left\{\mathcal{U}_{n}: n \in \omega\right\}$ de cubiertas abiertas de $X$ tal que $\bigcap\left\{s t^{k}\left(\mathcal{U}_{n}, x\right): n \in \omega\right\}=\{x\}$, para cada $x \in X$. Como $\sigma \subseteq \tau$, la familia $\left\{\mathcal{U}_{n}: n \in \omega\right\}$ es una sucesión de cubiertas abiertas de $G$ tal que $\bigcap\left\{s t^{k}\left(\mathcal{U}_{n}, x\right): n \in \omega\right\}=\{x\}$, para cada $x \in G$. Por lo tanto, $G$ tiene diagonal $G_{\delta}$ de rango infinito.

Un espacio $X$ es débilmente Lindelöf si para cada cubierta abierta $\mathcal{U}$ de $X$, existe $\lambda \subseteq \mathcal{U}$ tal que el conjunto $\bigcup \lambda$ es denso en $X$. Claramente, todo espacio Lindelöf es débilmente Lindelöf. En [27] se prueba que todo grupo paratopológico regular tiene índice de regularidad numerable. No sabemos si el teorema 3.3.2 se puede extender a grupos paratopológicos débilmente Lindelöf.

Problema 3.3.7. Sea $G$ un grupo paratopológico regular débilmente Lindelöf con pseudocarácter numerable. ¿Es G submetrizable?, ¿Existe una condensación de $X$ sobre un espacio Hausdorff segundo numerable?

Un espacio $X$ es Urysohn si para cada par de puntos distintos $x, y \in X$, existen vecindades $U$ y $V$ de $x$ y $y$, respectivamente, tales que $\bar{U} \cap \bar{V}=\emptyset$.

Teorema 3.3.8. Si $G$ es un grupo semitopológico regular $\omega$-estrecho tal que $H s(G) \psi(G) \leq \omega$, entonces existe una condensación de $G$ sobre un espacio Urysohn segundo numerable.

Demostración. Por hipótesis, existe una familia $\left\{V_{n}: n \in \omega\right\} \subseteq \mathcal{N}(e)$ la cual satisface $\bigcap_{n \in \omega} V_{n} V_{n}^{-1}=\{e\}$ y $\bar{V}_{n+1} \subseteq V_{n}$ para cada $n \in \omega$. Como $G$ es $\omega$ estrecho, para cada $n \in \omega$, existe $A_{n} \subseteq G$ numerable tal que $V_{n} A_{n}=G$. Sea $A=\bigcup_{n \in \omega} A_{n}$. Sea $\mathcal{P}=\left\{V_{n} a: n \in \omega, a \in A\right\} \cup\left\{G \backslash \overline{V_{n} a}: n \in \omega, a \in A\right\}$. Es claro que $\mathcal{P}$ es una familia numerable de abiertos en $G$. Sea $\sigma$ la topología en $G$ generada por $\mathcal{P}$. Probemos que $X=(G, \sigma)$ es un espacio Urysohn segundo 
numerable. Como $\mathcal{P}$ es numerable, sólo falta demostrar que $X$ es Urysohn. Como $V_{n} a$ y $G \backslash \overline{V_{n} a}$ son abiertos en $X$ tenemos que ${\overline{V_{n} a}}^{X}={\overline{V_{n} a}}^{G}=\bar{V}_{n}^{G} a$ y ${\overline{X \backslash \bar{V}_{n} a}}^{X} \subseteq X \backslash V_{n} a$ para cada $n \in \omega$ y $a \in A$. Tomemos una pareja $x, y \in G$ de puntos distintos. Como $\bigcap_{n \in \omega} V_{n} V_{n}^{-1}=\{e\}$, existe $n \in \omega$ tal que $y x^{-1} \notin V_{n} V_{n}^{-1}$. Podemos encontrar $a \in A$ tal que $x \in V_{n+2} a$. Si $y \in{\overline{V_{n+1} a}}^{X}$, entonces $y x^{-1} \in\left({\overline{V_{n+1} a}}^{X}\right)\left(a^{-1} V_{n+2}^{-1}\right)=\left(\bar{V}_{n+1}^{G} a\right)\left(a^{-1} V_{n+2}^{-1}\right)=\bar{V}_{n+1}^{G} V_{n+2}^{-1} \subseteq$ $V_{n} V_{n}^{-1}$, lo cual contradice la elección de $V_{n}$. Por lo tanto, $y \in X \backslash \overline{V_{n+1} a} X$ y $x \in V_{n+2} a$. Además, ${\overline{X \backslash{\overline{V_{n+1}}}^{X}}}^{X} \subseteq X \backslash V_{n+1} a$ y ${\overline{V_{n+2} a}}^{X}=\bar{V}_{n+2}^{G} a \subseteq V_{n+1} a$. Hemos demostrado que $X$ es un espacio Urysohn segundo numerable.

Corolario 3.3.9. Todo grupo semitopológico regular, primero numerable y $\omega$-estrecho se puede condensar sobre un espacio Urysohn segundo numerable.

En grupos paratopológicos podemos debililtar el axioma de separación en las hipótesis del teorema 3.3.8.

Corolario 3.3.10. Sea $G$ un grupo paratopológico Hausdorff $\omega$-estrecho el cual satisface $H s(G) \psi(G) \leq \omega$. Entonces existe una condensación de $G$ sobre un espacio Urysohn segundo numerable.

Demostración. Por la proposición 1.4.1, el grupo $r(G)$ tiene número de Hausdorff y pseudocarácter numerable. Además, como la topología de $r(G)$ es más débil que la topología de $G$, podemos concluir que $r(G)$ es $\omega$-estrecho. Por el teorema anterior el grupo paratopológico $r(G)$ se puede condensar sobre un espacio Urysohn segundo numerable. Esto termina la demostración.

Proposición 3.3.11. Si $G$ es un grupo paratopológico Hausdorff $\omega$-estrecho con $\pi$-carácter numerable, entonces $G$ admite una condensación sobre un espacio Urysohn segundo numerable.

Demostración. Por el lema 3.2.1 y la proposición 3.2.14, tenemos la desigualdad $H s(G) \psi(G) \leq \omega$. Para terminar basta aplicar el corolario 3.3.10.

En un intento de generalizar el teorema $3.3 .8 \mathrm{y}$ el corolario 3.3.10 se plantea la siguiente pregunta.

Problema 3.3.12. Sea $G$ es un grupo semitopológico Hausdorff $\omega$-estrecho con $H s(G) \psi(G) \leq \omega$. ¿Existe una condensación de $G$ sobre un espacio Hausdorff segundo numerable? 


\section{Capítulo 4}

\section{Conclusiones}

A lo largo de este trabajo se obtuvieron varios resultados en forma positiva y algunos contraejemplos que respondían varias preguntas planteadas en nuestro proyecto de trabajo. Cabe resaltar que se responden algunos problemas publicados por matemáticos como A. Arhangel'skii, A. Bella, M. Sanchis y M. Tkachenko. Sin embargo, no todas las preguntas consideradas en nuestro proyecto fueron resueltas. En la siguiente sección mencionamos algunos problemas, que hasta donde este autor sabe, todavía siguen sin responderse.

\subsection{Problemas abiertos}

En un intento de extender la proposición 2.1.5 a grupos semitopológicos se plantea el siguiente problema.

Problema 4.1.1. ¿Cada grupo semitopológico regular $G$ cumple la desigual$\operatorname{dad} \operatorname{Sm}(G) \leq l(G)$ ?

Por $[32$, Theorems $2.7,3.6]$ y nuestros teoremas 2.2 .8 y 2.2.11, los grupos paratopológicos proyectivamente $T_{i}$ primero numerables están caracterizados internamente, para $i=0,1,2,3$.

Problema 4.1.2. Dar una caracterización interna de los grupos paratopológicos proyectivamente $T_{3 \frac{1}{2}}$ primero numerables.

A partir de las definiciones de número de Hausdorff y número de simetría se puede concluir que para un grupo paratopológico Hausdorff $G$ se tiene $\operatorname{Sm}(G) \leq H s(G)$. Si se satisface la desigualdad $H s(G) \leq \omega$, entonces 
$\operatorname{Sm}(G)=H s(G)$. Denotemos por $\mathcal{C}_{\omega}$ a la clase de grupos paratopológicos Hausdorff que cumplen $H s(G) \leq \omega$. Por las proposiciones 2.1-2.4 en [32], $\mathcal{C}_{\omega}$ contiene a los grupos paratopológicos Hausdorff primero numerables, Lindelöf, grupos topológicos, la clase $\mathcal{C}_{\omega}$ es cerrada bajo subgrupos y productos arbitrarios. En general, la igualdad $\operatorname{Sm}(H)=H s(H)$ no se cumple para un grupo paratopológico $H$. Basta tomar el grupo $H$ del ejemplo 2.2.23. Con base en las observaciones anteriores surge la siguiente pregunta.

Problema 4.1.3. ¿Qué propiedades debe tener un grupo paratopológico $G$ para que se satisfaga la igualdad $\operatorname{Sm}(G)=H s(G)$ ?

Por el teorema 3.1.3, cada grupo paratopológico regular totalmente $\omega$ estrecho tiene índice de regularidad numerable. También por el corolario 3.1.14, cada grupo paratopológico regular precompacto tiene índice de regularidad numerable. Estos resultados sugieren la siguiente pregunta.

Problema 4.1.4. ¿Cada grupo paratopológico regular $\omega$-estrecho tiene índice de regularidad numerable?

En un intento de generalizar a grupos semitopológicos las proposiciones 3.2 .13 y 3.2.14, se plantea la siguiente cuestión:

Problema 4.1.5. Sea $G$ un grupo semitopológico regular con $\pi$-carácter numerable. ¿Tiene $G$ indice de regularidad (número de Hausdorff) numerable?

Es claro que todo grupo semitopológico primero numerable es $\omega$-balanceado. Esto junto con los resultados de la sección 3.2 motivan la siguiente interrogante.

Problema 4.1.6. ¿ ¿Todo grupo paratopológico Hausdorff (regular) con $\pi$ carácter numerable es $\omega$-balanceado?

No sabemos si el teorema 3.3.2 se puede extender a grupos paratopológicos débilmente Lindelöf.

Problema 4.1.7. Sea $G$ un grupo paratopológico regular débilmente Lindelöf con pseudocarácter numerable. ¿Es $G$ submetrizable?, ¿Existe una condensación de $X$ sobre un espacio Hausdorff segundo numerable?

En un intento de generalizar el teorema 3.3.8 y el corolario 3.3.10 se plantea la siguiente pregunta.

Problema 4.1.8. Sea $G$ un grupo semitopológico Hausdorff $\omega$-estrecho con $H s(G) \psi(G) \leq \omega$. ¿Existe una condensación de $G$ sobre un espacio Hausdorff segundo numerable? 


\section{Bibliografía}

[1] O.T. Alas, M. Sanchis, Countably compact paratopological groups, Semigroup Forum 74 (2007) 423-438.

[2] A. V. Arhangel'skii, A. Bella, The diagonal of a first countable paratopological group, submetrizability, and related results, Applied General Topology, Volume 8, No. 2 (2007), 207-212.

[3] A. V. Arhangel'skii, D. K. Burke, Spaces with a regular $G_{\delta}$-diagonal, Topol. Appl. 153 No. 11 (2006), 1917-1929.

[4] A.V. Arhangel'skii, M.M. Choban, P.S. Kenderov, Topological games and continuity of groups operations, Topology Appl. 157 (2010) 25422552 .

[5] A.V. Arhangel'skii, E.A. Reznichenko, Paratopological and semitopological groups versus topological groups, Topology Appl. 151 (2005) $107-119$.

[6] A.V. Arhangel'skii, M.G. Tkachenko, Topological Groups and Related Structures, Atlantis Studies in Mathematics, Vol. I, Atlantis Press/World Scientific, Paris-Amsterdam, 2008.

[7] T. Banakh, A. Ravsky, On subgroups of saturated or totally bounded paratopological groups, Algebra and Discrete Mathematics No. 4 (2003) 1-20.

[8] T. Banakh, O. Ravsky, Oscillator topologies on a paratopological group and related number invariants, Algebraical Structures and their Applications, Kyiv: Inst. Mat. NANU (2002), 140-153. 
[9] D. Basile, A. Bella, G.J. Ridderbos, Weak extent, submetrizability and diagonal degrees, http://arxiv.org/abs/1112.0883 (December 5, 2011).

[10] N. Brand, Another note on the continuity of the inverse, Arch. Math. 39 (1982) 241-245.

[11] A. Bouziad, Every Cech-analytic Baire semitopological group is a topological group, Proc. Amer. Math. Soc. 124 (1996) 953-959.

[12] R. Z. Buzyakova, Observations on spaces with zeroset or regular $G_{\delta^{-}}$ diagonals, Comment. Math. Univ. Carolin. 46 (2005), No. 3, 469-473.

[13] R. Ellis, Locally compact transformation groups, Duke Math. J. 24 (1957) 119-125.

[14] R. Engelkig, General Topology, Heldermann Verlag, Berlin, 1989.

[15] I. Guran, Topological groups close to being Lindelöf, Soviet Math. Dokl. 23 (1981) 173-175; Russian original in: Dokl. Akad. Nauk SSSR 256 (1981) 1035-1037.

[16] E. Hewitt and K. A. Ross, Abstract Harmonic Analysis. Vol. I, Structure of Topological Groups, Integration Theory, Group Representations. Second edition. Fund. Prin. of Math. Sci., 115. (Springer-Verlag, BerlinNew York, 1979).

[17] I. Juhász, Cardinal Functions in Topology-Ten Years Later, second ed., Mathematical Centre Tracts, vol. 123, Mathematisch Centrum, Amsterdam, 1980.

[18] G.I. Katz, Isomorphic mapping of topological groups to direct product of groups satisfying the first axiom of countability, Uspekhi Mat. Nauk 8 (1953) 107-113 (in Russian).

[19] P.S. Kenderov, I.S Kortezov, W.B. Moors, Topological games and topological groups, Topology Appl. 109 (2001) 157-165.

[20] P. Li, L. Mou, S. Wang, Notes on questions about spaces with algebraic structures, Topology Appl. 159 (2012), 3619-3623. 
[21] C. Liu, A note on paratopological groups, Comment. Math. Univ. Carolin. 47 No. 4 (2006), 633-640.

[22] D. Montgomery, Continuity in topological groups, Bull. Amer. Math. Soc. 42 (1936) 879-882.

[23] L.S. Pontryagin, Continuous Groups, third edition, "Nauka", Moscow 1973.

[24] O.V. Ravsky, (2-)Pseudocompact paratopological groups that are topological, http://arxiv.org/abs/1003.5343 (28 de Marzo de 2010).

[25] O.V. Ravsky, Paratopological groups I, Matematychni Studii 16 (2001), No. 1, 37-48.

[26] O.V. Ravsky, Paratopological groups II, Matematychni Studii 17 (2002), No. 1, 93-101.

[27] I. Sánchez, Cardinal invariants of paratopological groups, Topological Algebra and its Applications (2013) 37-45.

[28] I. Sánchez, Subgroups of products of paratopological groups, Topology Appl. (2013), http://dx.doi.org/10.1016/j.topol.2013.10.015

[29] M. Sanchis, M. G. Tkachenko, Recent progress in paratopological groups, Quaderni Math. (2012), in press.

[30] M. Sanchis, M.G. Tkachenko, Totally Lindelöf and totally w-narrow paratopological groups, Topology Appl. 155 (2007) 322-334.

[31] M.G. Tkachenko, Introduction to topological groups, Topology Appl. 86 (1998) 179-231.

[32] M.G. Tkachenko, Embedding paratopological groups into topological products, Topology Appl. 156 (2009) 1298-1305.

[33] M.G Tkachenko, Paratopological and semitopological groups vs topological groups, In: Recent Progress in General Topology III, Elsevier, to appear. 
[34] M. G. Tkachenko, Paratopological Groups: Some Questions and Problems, Q\&A in General Topology 27 No. 1 (2009), 1-21.

[35] W. Żelazko, A theorem on $B_{0}$ division algebras, Bull. Acad. Pol. Sci. 8 (1960) 373-375.

[36] P. Zenor, On spaces with regular $G_{\delta}$-diagonals, Pacific J. Math. 40 (1972), 759-763. 